\title{
One for "All": A unified model for fine-grained sentiment analysis under three tasks
}

\author{
Heng-yang Lu ${ }^{1,2}$, Jun Yang ${ }^{\text {Corresp., }}{ }^{,}$, Cong Hu ${ }^{1}$, Wei Fang ${ }^{1}$ \\ 1 Jiangsu Provincial Engineering Laboratory of Pattern Recognition and Computational Intelligence, Jiangnan University, Wuxi, China \\ 2 State Key Laboratory for Novel Software Technology, Nanjing University, Nanjing, China \\ 3 Marcpoint Co., Ltd., Shanghai, China \\ Corresponding Author: Jun Yang \\ Email address: jun.yang@marcpoint.com
}

Background. Fine-grained sentiment analysis is used to interpret consumers' sentiments, from their written comments, towards specific entities on specific aspects. Previous researchers have introduced three main tasks in this field (ABSA, TABSA, MEABSA), covering all kinds of social media data (e.g. review specific, questions and answers, and community-based). In this paper, we identify and address two common challenges encountered in these three tasks, including the low-resource problem and the sentiment polarity bias.

Methods. We propose a unified model called PEA by integrating data augmentation methodology with the pre-trained language model, which is suitable for all the ABSA, TABSA and MEABSA tasks. Two data augmentation methods, which are entity replacement and dual noise injection, are introduced to solve both challenges at the same time. An ensemble method is also introduced to incorporate the results of the basic RNNs-based and BERT-based models.

Results. PEA shows significant improvements on all three fine-grained sentiment analysis tasks when compared with state-of-the-art models. It also achieves comparable results with what the baseline models obtain while using only $20 \%$ of their training data, which demonstrates its extraordinary performance under extreme low-resource conditions. 


\section{One for "All": A unified model for fine-grained} 2 sentiment analysis under three tasks

3

4

5 6

7

8

Heng-yang $\mathrm{Lu}^{1,2}$, Jun Yang ${ }^{3, *}$, Cong $\mathrm{Hu}^{1}$, Wei Fang ${ }^{1}$

${ }^{1}$ Jiangsu Provincial Engineering Laboratory of Pattern Recognition and Computational Intelligence, Jiangnan University, Wuxi, Jiangsu, China.

${ }^{2}$ State Key Laboratory for Novel Software Technology, Nanjing University, Nanjing, Jiangsu, China.

${ }^{3}$ Marcpoint Co., Ltd., Shanghai, China.

Corresponding Author:

Jun Yang

No. 456, Hongcao Road, Xuhui District, Shanghai, 200233, China

Email address: jun.yang@marcpoint.com 


\section{Abstract}

43 Background. Fine-grained sentiment analysis is used to interpret consumers' sentiments, from

44

45

46

47

48

49

50

51

52

53

54

55

56

57

58

\section{9}

60

61

62

63

64

65

66

67

68

69

70

71

72

73

74

75

76

77

78

79

80

81

their written comments, towards specific entities on specific aspects. Previous researchers have introduced three main tasks in this field (ABSA, TABSA, MEABSA), covering all kinds of social media data (e.g. review specific, questions and answers, and community-based). In this paper, we identify and address two common challenges encountered in these three tasks, including the low-resource problem and the sentiment polarity bias.

Methods. We propose a unified model called PEA by integrating data augmentation methodology with the pre-trained language model, which is suitable for all the ABSA, TABSA and MEABSA tasks. Two data augmentation methods, which are entity replacement and dual noise injection, are introduced to solve both challenges at the same time. An ensemble method is also introduced to incorporate the results of the basic RNNs-based and BERT-based models.

Results. PEA shows significant improvements on all three fine-grained sentiment analysis tasks when compared with state-of-the-art models. It also achieves comparable results with what the baseline models obtain while using only $20 \%$ of their training data, which demonstrates its extraordinary performance under extreme low-resource conditions.

\section{Introduction}

Consumers worldwide have posted trillions of text comments on online shopping sites and social platforms to express their opinions. The efficiency of how modern merchandisers drive insights from those opinions would be the key to their success in the data-driven era. Sentiment analysis is such a solution for businesses to understand consumers' opinions effectively. Traditional coarse-grained sentiment analysis aims to identify the sentiment polarity of the given sentence. Different from that, fine-grained sentiment analysis is managed to match sentiments with corresponding entities and aspects in the given sentence. For example, given the comment "I've used MacBookPro, it's convenient." Coarse-grained sentiment analysis describes the whole sentence a positive sentiment. Fine-grained sentiment analysis describes a positive sentiment towards MacBookPro (entity) on its convenience level (aspect), which is a provided (sentence, aspect, entity) pair. Previous researchers have introduced three tasks on fine-grained sentiment analysis towards entities and aspects (definitions and 2 examples are illustrated in Table 1) :

1. Aspect-Based Sentiment Analysis (ABSA),

2. Targeted Aspect-Based Sentiment Analysis (TABSA),

3. Multi-Entity Aspect-Based Sentiment Analysis (MEABSA).

ABSA was primarily based on the review-specific data acquired from E-commerce or life service websites (e.g. Amazon, Yelp) where there is only one or even no entity mentioned in the data. Although performing well on consumer reviews, models designed for ABSA have limited performance on posts coming from social platforms (e.g. Twitter, Reddit) where there are multiple entities and aspects mentioned. For example, a software engineer on Twitter wrote "I've used MacBookPro, it's convenient. But now I switched to ThinkPad because it's just as convenient and has a better price." There are two entities introduced: MacBookPro and 
82 ThinkPad. For each of the entities, sentiments on the convenience level (aspect 1) are the same 83 while sentiments on the price (aspect 2) are different. TABSA was proposed by (Saeidi et al., 84 2016) to handle such multi-entity and multi-aspect cases. This task was based on the SentiHood

85

86

87

88

89

90

91

92

93

94

95

96

97

98

99

100

101

102

103

104

105

106

107

108

109

110

111

112

113

114

115

116

117

118

119

120

121

122 dataset acquired from the question answering platform, which involves 2 entities of the same kind (e.g. tourist attractions) and 15 aspects. However, in reality, not only do consumers compare entities of that same kind but also should they talk about multi-kind entities. Yang (Yang et al., 2018) proposed MEABSA with the BabyCare dataset acquired from a community-based platform. It involves hundreds of multi-kind entities (e.g. powdered milk, diapers, and infant medicines) and hundreds of aspects. The increase in the number of entities and aspects makes MEABSA the most challenging task among the three.

Most previous works are designed for only one of the tasks, it is more practical to design a unified model, which is available for all three tasks. What's more, the Recurrent Neural Network (RNN)-based models (Yang et al., 2018; Yang et al., 2019; Xu et al., 2020) and BERT-based models (Sun, Huang \& Qiu, 2019) are two kinds of recently proposed basic models for finegrained sentiment analysis, which have shown effectiveness. The RNN-based models have the advantages of considering the global sequence, and the BERT-based models are good at considering local attention. It is promising to improve the predictions of sentiments by making use of both advantages.

Additionally, there are two main challenges encountered in the ABSA, TABSA, and MEABSA tasks. The first challenge is the low-resource problem, also known as the insufficient data problem. This is often caused by the large time and money required by manual annotation. The low-resource problem is even more prevalent in sentiment prediction towards entities and aspects due to the increasing complexity of data annotation: for example, if there are 3 entities and 2 aspects mentioned in the text, one needs to annotate $6(3 * 2)$ instances for each of the entity aspect combinations. This explains the fact that $59 \%$ of the entity aspect combinations are annotated 5 times or less in the BabyCare dataset. The second challenge is the polarity bias problem. It reduces task performance when entities' sentiment polarity distribution is not uniform in the training set. For example, if an entity is mostly labeled positive in the training set, it will be more likely to be predicted positive regardless of the context. This problem is mainly caused by the inconsistent polarity distributions between the training set and test set from the perspective of entities.

This paper aims to propose a unified model for fine-grained sentiment analysis, which is available for ABSA, TABSA and MEABSA tasks. The main contributions of this paper include:

- To the best of our knowledge, this is the first work unifying the ABSA, TABSA, and MEABSA tasks together, providing an all-in-one solution to fine-grained sentiment analysis.

- We propose a unified model, which combines both advantages of RNN-based models and BERT-based models with ensemble methods. This model achieves outstanding performance in all the ABSA, TABSA, and MEABSA tasks.

- This paper considers the low-resource and polarity bias problems in the fine-grained sentiment analysis for the first time. Two data augmentation methods include entity replacement and noise injection are designed to deal with the problems. 


\section{Literature Reviews}

125

126

127

128

129

130

131

132

133

134

135

136

137

138

139

140

141

142

143

144

145

146

147

148

149

150

151

152

153

154

155

156

157

158

159

160

161

\section{Research on fine-grained sentiment analysis}

There are abundant researches on the ABSA task. LSTM (Tang et al., 2016) and an attention mechanism (Wang et al., 2016) have been applied to deal with the ABSA task in early time. Following works include applying memory network-based (Tang, Qin \& Liu, 2016) and attention-based (Chen et al., 2017) method to LSTM models, involving two stacked LSTMs (Xu et al., 2020) and so on. More recent models such as capsule network (Chen \& Qian, 2019; Du et al., 2019), graph convolutional network model (Zhang, Li \& Song, 2019), graph attention network (Wang et al., 2020), bi-level interactive graph convolution network (Zhang and Qian, 2020) are also used for ABSA task. Zhu and associates have exploited the interaction between the aspect category and the contents under the guidance of both sentiment polarity and predefined categories, and the proposed aspect aware learning framework has achieved satisfying performance in ABSA (Zhu et al., 2019). The interactive relationships among aspect term extraction, opinion term extraction, and aspect-level sentiment classification have been investigated to encode collaborative signals for unified ABSA tasks (Chen and Qian, 2020). The pre-trained model such as RoBERTa has also been applied to improve ABSA with induced trees (Dai et al., 2021).

Saeidi and associates (Saeidi et al., 2016) first proposed the TABSA task with SentiHood dataset. Following works include using additional commonsense knowledge (Ma, Peng \& Cambria, 2018), developing a delayed memory update mechanism (Liu, Cohn \& Baldwin, 2018), extending LSTM by adding the external knowledge (Khine et al., 2019) and so on. Additionally, Ye and Li proposed a recurrent entity memory network with word-level information and sentence-level hidden memory for TABSA (Ye and Li, 2020). In recent years, pre-trained language model is also applied to capture the dependence on both targets and aspects for sentiment prediction (Wan et al., 2020). BERT model has been applied to TABSA task. For example, auxiliary sentence has been found useful in TABSA when BERT model is applied (Sun, Huang \& Qiu, 2019). Similarly, Hong and associates (Hong \& Song, 2020) further finetune the pre-trained BERT model on SentiHood dataset. What's more, a context-guided softmaxattention and context-guided quasi-attention method is proposed to perform aspect categorization and TABSA at the same time (Wu and Ong, 2020).

Yang and associates (Yang et al., 2018) first proposed the MEABSA task and contributed a dataset named BabyCare. They also proposed the Context memory, Entity memory and Aspect memory model (CEA) with RNN and deep memory networks. To improve the performance on long and complex text, an extended model of combining dependency trees with deep neural networks was proposed (Yang et al., 2019). The data sparsity challenge, also known as the cold-start problem, has also been investigated in MEABSA, which designed the frequency-guided attention mechanism to solve the problem (Song et al.,2019).

Research on data augmentation in NLP

Peer) Comput. Sci. reviewing PDF | (CS-2021:10:66426:1:0:NEW 14 Nov 2021) 
162 To alleviate the low-resource problem in various NLP tasks, data augmentations have been

163

164

165

166

167

168

169

170

171

172

173

174

175

176

177

178

179

180

181

182

183

184

185

186

187

188

189

190

191

192

193

194

195

196

197

198

199

200

201

applied in previous works. The optional strategies mainly include word replacement, noise injection, text generation and so on. For example, it is useful to generate additional training examples that contain rare words in synthetically created contexts for machine translation (Fadaee, Bisazza \& Monz, 2017). Another similar idea injected low-resource words into highresource sentences to improve the low-resource translation task (Xia et al., 2019). Additionally, data augmentations such as synonym replacement and delexicalization have been applied to the NER task (Dai \& Adele, 2020) and dialogue language understanding (Hou et al., 2018) respectively. Kim and associates (Kim, Roh \& Kim, 2019) proposed a method for spoken language understanding by introducing noise in all slots without classifying types of slots to improve the performance of low-resource dataset with "open-vocabulary" slots.

\section{Research on Bias problems in NLP}

Bias, such as racial bias and gender bias (Kiritchenko \& Mohammad, 2018; Thelwall, 2018), is also a trending topic of concern in different NLP researches. For example, Zhao and associates (Zhao et al., 2018) tried to mitigate gender bias by creating an augmented dataset identical to the original one by replacing the entities such as "he" or "she". Another work formally proposed the Counterfactual data augmentation (CDA) for gender bias mitigation in the coreference resolution task, by replacing every occurrence of a gendered word in the original corpus with its flipped one (Lu et al., 2020).

Recently, there are some related works to deal with the low-resource and polarity bias problems in coarse-grained sentiment analysis, which aims to predict the sentiments of the given posts. An early work introduced a bias-aware thresholding method motivated by cost-sensitive learning (Iqbal, Karim \& Kamiran, 2015). Recent works include designing a sentiment bias processing strategy for the lexicon-based sentiment analysis (Han et al., 2018), and using the generationbased data augmentation method to deal with the low-resource problem in coarse-grained sentiment analysis (Gupta, 2019). To the best of our knowledge, there is no recent work discussing solutions to low-resource or polarity bias problems in fine-grained sentiment analysis.

\section{Methods}

ABSA, TABSA and MEABSA are three widely discussed tasks for fine-grained sentiment analysis, whose common objective is to predict the sentiment towards each aspect of each target entity. The detailed comparisons and examples can be found in Table 1 in the introduction section. This section introduces the methodologies, which we used to unify the ABSA, TABSA, and MEABSA tasks together with the same architecture. The proposed all-in-one solution to Predict sentiment towards Entities and Aspects is named PEA. Fig. 1 demonstrates the graphical abstract of the PEA model.

Firstly, the unified problem setting of fine-grained sentiment analysis covering ABSA, TABSA and MEABSA is as follows.

\section{Problem Setting}

Peer] Comput. Sci. reviewing PDF | (CS-2021:10:66426:1:0:NEW 14 Nov 2021) 
202 Given a post Post $_{m}=\left[w_{1}, w_{2}, \ldots, w_{T}\right]$, with an entity set (if available) $E_{m}=$ entity $_{1}$, entity en $_{2}$ $203, \ldots$, entity $\left._{\left|E_{m}\right|}\right\}$ and an aspect set $A_{m}=\left\{\right.$ aspect $_{1}$, aspect ${ }_{2}, \ldots$, aspect $\left._{\left|A_{m}\right|}\right\}$. For the words or 204 multiple words in Post $_{m}$, which are corresponding to the entities or aspects in $E_{m}$ or $A_{m}$, we call 205 them entity terms and aspect terms. The fine-grained sentiment analysis aims to predict the 206 sentiment $y_{\text {entity }_{i}}^{\text {aspect }_{j}}$ towards the given aspect $j_{j}$ of the certain entity $_{i}$ in $P_{m}$.

207 For the ABSA task, the entity set $E_{m}=\varnothing$ and the prediction target is simplified to $y^{\text {aspect }_{j}}$. 208 For the TABSA task, in each post Post ${ }_{m}$, there is only one or two entities in the entity set, where $209\left|E_{m}\right|=1$ or $\left|E_{m}\right|=2$. The prediction target becomes $y_{\text {entity }_{i}}{ }_{j}$ towards all the aspects for the 210 target entity in Post $_{m}$.

211 For MEABSA, the most challenging task, there are multiple entities and aspects in Post $_{m}$, where

$212\left|E_{m}\right| \geq 1$ and $\left|A_{m}\right| \geq 1$. It aims to predict $y_{\text {entity }_{i}}^{\text {aspect }_{j}}$ towards the mentioned aspects for every entity 213 entity $_{i}$ in Post $_{m}$.

214 The general training workflow of PEA includes:

215 (1) given an original training set $D$, generate a new training set $D$ ' based on entity replacement.

216 For the ABSA task, there is no entity involved, so the entity replacement step is skipped and $D^{\prime}$

$217=D$. For TABSA and MEABSA, entity replacement is conducted to get an entity-replaced 218 dataset $P D$, and $D^{\prime}=D \cup P D$. The entity replacement used in PEA is introduced in the first part 219 of subsection "Data Augmentation".

220 (2) An RNN-based model is trained on the new training set $D$ ' as one of the basic models. The 221 dual noise injection is conducted on the input posts, entities and aspects to get the noise-injected 222 vectors. The dual injection used in PEA is introduced in the second part of subsection "Data 223 Augmentation". Then, we take an attentional recurrent neural network-based model, CEA (Yang 224 et al., 2018), as an example, to be the basic model, whose output is the predicted sentiment 225 polarity distribution of the given inputs. It is introduced in the first part of subsection "Basic 226 Models".

227 (3) A pre-trained language model is trained on the new training set $D^{\prime}$ as the other basic model. 228 Auxiliary question sentences are constructed for training the BERT-based model, which can 229 predict fine-grained sentiment polarity distribution with the given inputs. The detailed design is 230 described in the second part of subsection "Basic Models".

231 (4) Finally, the ensemble method is applied to fuse the predicted sentiment polarity distribution 232 of the RNN-based and BERT-based model as the outputs of PEA, which is the final predicted 233 sentiment polarity. The fusion strategy is introduced in the third part of subsection "Fusion 234 Strategy".

235 Data Augmentation 
236 Data augmentation is widely used to improve learning performance, prevent overfitting, and

237

238

239

240

241

242

243

244

245

246

247

248

249

250

251

252

253

254

255

256

257

258

259

260

261

262

263

264

265

266

267

268

269

270

271

272

273

increase robustness under low-resource conditions. This section illustrates two innovative, taskspecific data augmentation methods that are deployed in the model.

Entity Replacement. The low-resource problem in fine-grained sentiment analysis mainly comes from entities in the posts. This problem can be alleviated by increasing the low-resource entities. Among the data augmentation methods used in recent works for alleviating the lowresource problem in other NLP tasks, replacing words in context with similar ones is a viable data augmentation method (Fadaee, Bisazza \& Monz, 2017; Xian et al., 2019; Dai \& Adel, 2020). Usually, similar words can be extracted from word similarity calculation (Wang \&Yang, 2015), and can also be extracted from a handcraft ontology such as WordNet.

In previous works, any word in a sentence can be replaced. This kind of replacement is extremely risky in fine-grained sentiment analysis tasks. For example, if a sentiment word, such as "happy", was replaced, it would unintentionally change the sentiment polarity at the same time. To avoid this kind of situation, we proposed the entity replacement method which successfully addresses this problem. Entity replacement is used to generate pseudo instances for training. The entire process involves 3 steps:

- $\quad$ Creating a duplicate of the original training set $D$.

- Replacing each entity in the duplicated dataset with the target entity to get an entity-replaced dataset $P D$.

- Combining the original dataset with the entity-replaced dataset as the new training dataset $D^{\prime}$ $=D \cup P D$ to train models.

In step 2, target entities are selected dynamically based on the scarcity of entities in the original training set so that every entity will have sufficient training instances eventually. In other words, the fewer times an entity presents in the original training set, the more likely it will be selected as the target entity. The detailed probability that an entity is selected is calculated as follows:

$$
\mathrm{P}\left(\text { entity }_{i}\right)=\frac{\mid \text { mention }\left.\left(\text { entity }_{i}\right)\right|^{-1}}{\sum_{j=1}^{|E|} \mid \text { mention }\left.\left(\text { entity }_{j}\right)\right|^{-1}}, \forall i \in[1,|E|]
$$

where $E=\bigcup_{E_{m} \in D} E_{m}$ is the total entity set in the original training dataset $D$, $\mid$ mention $\left(\right.$ entity $\left._{i}\right) \mid$ represents the number of instances mentioning entity $y_{i}$ in $D . x^{-1}$ is an inverse proportional function, where $x^{-1}=\frac{1}{x}$.

Table 2 shows an example of such a replacement. Besides increasing the number of training instances, we think data augmentation also helps solve the polarity bias problem. For example, if an entity is always labeled positive in the training set, it will be more likely to be predicted positive no matter what the post is about. The proposed data augmentation methods help the polarity balance for entities because the entity may be replaced into a positive or neutral or negative expression randomly.

To conclude, the low-resource entity replacement is designed to increase the number of training instances, especially for the low-resource entities, and help solve the polarity bias problem in sentiment prediction towards multiple entity settings.

Peer] Comput. Sci. reviewing PDF | (CS-2021:10:66426:1:0:NEW 14 Nov 2021) 
274 Dual Noise Injection. To improve the generalization ability of PEA, we also involve the noise 275 injection method. In previous NLP tasks, such as machine translation (Cheng et al., 2018) and 276 spoken language understanding (Kim, Roh \& Kim, 2019), it has shown the effectiveness of 277 improving the model's generalization ability by injecting noises. In these works, noise is usually 278 injected into the context representation for the post directly. For fine-grained sentiment analysis, 279 the inputs include context texts, entities, entity terms, aspects and aspect terms. It is not 280 applicable to only inject noises on context representations like previous works. Therefore, we 281 propose the idea of dual noise injection: a noise is injected into the representation of entity and 282 entity terms in the context at the same time. A similar practice is performed on the aspect and 283 aspect terms.

284 In this task, the dual noise injection is used to simulate new entities and new aspects, enabling 285 the model to make better predictions when it comes across low-resource entities or aspects.

286 Following the common choice of previous works (Cheng et al., 2018; Kim, Roh \& Kim, 2019), 287 we also use the Gaussian noise to inject noises into the embedding space of posts, entities and 288 aspects. Fig. 2 is an example to illustrate the detailed processes of dual noise injection.

289 The dual noise injection consists of 3 steps:

290 - We first express the post, entity, and aspect in vectors space $v_{w} \in \mathbb{R}^{T \times k}, v_{e} \in \mathbb{R}^{k}, v_{a} \in \mathbb{R}^{k}$, 291 where $v_{w}=\left\{v_{w_{1}}, \ldots, v_{w_{T}}\right\}, T$ represents the number of words in the post, and $k$ is the dimension of representations. The embedding vectors can be initialized by GloVe (Pennington, Socher \& Manning, 2014).

294

- Then we sample noise vectors $n_{e} \in \mathbb{R}^{k}$ and $n_{a} \in \mathbb{R}^{k}$ for entity and aspect respectively from the Gaussian distribution.

- At last, we extract indicator vector $i_{e}=\left\{i_{e}^{0}, \ldots, i_{e}^{T}\right\}$ for entity terms indicating the location of entity terms in the post. Each element in $i_{e}$ is binary. $i_{e}^{t}$ is set to 1 when the $t^{t h}$ word in the post is an entity term, otherwise, it is set to 0 . Note that an entity term may consist of one or more words. In the same manner, we can get an indicator vector $i_{a}$ for aspect term. Then, we inject the noise to the entity, the aspect, and the post:

$$
\begin{gathered}
v_{e}^{\prime}=v_{e}+n_{\mathrm{e}^{\prime}} \\
v_{a}^{\prime}=v_{a}+n_{\mathrm{a} .} \\
v_{w_{i}}^{\prime}=v_{w_{i}}+i^{e} \times n_{\mathrm{e}}+i^{a} \times n_{\mathrm{a} .}
\end{gathered}
$$

In step 2, the same noise vector (e.g. $n_{\mathrm{e}}$ ) needs to be applied to the entity and entity term. This is to ensure the new-generated entity and entity term remain the same relative location in the embedding space. We also apply the same noise vector (e.g. $n_{\mathrm{a}}$ ) to the aspect and the aspect term in the same manner. The noise injected into the entity and aspect does not have to be equal. Also, if the noise level is not large enough, it won't substantially change the effect of injections. In order to test what is the best noise level in this case, we conduct experiments to determine the settings, which is introduced in section "Experimental Settings".

\section{Basic Models}


312 Recently, both RNN-based models and BERT-based models have shown effectiveness in the 313 fine-grained sentiment analysis (Yang et al., 2018; Yang et al., 2019; Sun, Huang \& Qiu, 2019; 314 Xu et al., 2020;). Due to the different structures of RNN and BERT, both kinds of models have 315 advantages and weaknesses respectively. PEA incorporates both models to help make the final 316 prediction more accurate.

317 RNN-based Model for Fine-grained Sentiment Analysis. The CEA model is designed for 318 MEABSA task, and can also be used for ABSA and TABSA tasks. It takes the word vectors of 319 the post, the entity vectors and aspect vectors as inputs, and predicts the fine-grained sentiments 320 towards the given aspect of the entity. To incorporate noise injection with CEA, we feed the 321 noise-injected vectors to CEA, the general structure of noise-injected CEA is as Fig. 3 shows. 322 Firstly, we feed every noise-injected word vector $v_{w_{i}}^{\prime}$ in the post to CEA. An LSTM layer is 323 applied to extract the semantics of the post after a few data processing layers. After that, a deep 324 memory network is applied to update entity and aspect representations with the given noise325 injected entity vector $v_{e}^{\prime}$ and aspect vector $v_{a}^{\prime}$. The updated representations are fed into a dense 326 layer to predict the final sentiment. For detailed explanation of CEA, refer to the original paper 327 (Yang et al., 2018).

328 Because CEA requires entities and aspects as inputs, it is naturally suitable for the TABSA and MEABSA tasks. For the ABSA task, if there is no entity mentioned in the post, we can set the entity vector to a zero vector as the input. This makes the CEA-based basic model be able to deal with all the ABSA, TABSA and MEABSA tasks. model is useful for enabling low-resource tasks to benefit from a huge amount of unlabeled data by pre-training. Bidirectional Encoder Representations from Transformers (BERT) (Devlin et al., 2018) is one of the key innovations in language representation learning (Howard \& Ruder, 2018; Peters et al., 2018). It has achieved good results in many natural language processing tasks (Acheampong et al., 2021; van Aken et al., 2019). BERT uses bidirectional pre-training for language representations, and it is pre-trained on two tasks: masked language model for understanding the relationship between words, and next sentence prediction for understanding the relationship between sentences for downstream tasks. The design of pre-training makes use of a huge amount of unlabeled data, making it suitable for low-resource situations. Thus, we incorporate BERT to further enhance performance. Sun (Sun, Huang \& Qiu, 2019) argued that constructing an auxiliary question sentence for the BERT model is useful in the TABSA task. We follow the conclusion and make the auxiliary question sentence for the entity and aspect with the template of "What is the sentiment towards the [aspect] of [entity]?". Then the sentiment classification task is turned into a sentence pair classification task. The label set of this setting includes \{Positive, Neutral, Negative\}. The BERT model takes two paragraphs as input with the token [CLS] at the beginning and [SEP] at the end of each paragraph. We set the post as the first paragraph and the auxiliary question sentence as the second. Here is an example.

Input: [CLS] I've used MacBookPro, it's convenient. [SEP] What is the 


\section{sentiment towards the convenience of MacBookPro? [SEP] \\ Output: Positive}

351 By constructing auxiliary question sentences along with the posts, we can generate inputs

352

353

354

355

356

357

358

359

360

361

362

363

364

365

366

367

368

369

370

371

372

373

374

375

376

377

378

379

380

381

382

383

384 suitable for training BERT-based models, whose outputs are the predictions of sentiments towards targeted aspects of entities.

The construction of inputs can be applied to the TABSA and MEABSA directly. For the ABSA task, there is no entity mentioned in the post, the underlined part in the constructed question template, which is "What is the sentiment towards the [aspect] of [entity]?", will be omitted. This makes the BERT-based basic model be able to deal with all the ABSA, TABSA and MEABSA tasks.

\section{Fusion Strategy}

Ensemble methods can improve the predictive performance of a single model by training multiple models and combining their predictions. The weighting method is one of the effective strategies to fuse outputs, which assign weights to each basic model to combine the final decision (Sagi \& Rokach, 2018), including simple averaging and weighted averaging (Zhou, 2021). We follow the strategy of simple averaging and combine the data augmented CEA with BERT to be the final model. We train the two models separately, and ensemble their predictions by taking the sentiment polarity with the largest averaged predicted probability as the final output. For a given post Post $_{m}$, the fine-grained sentiment prediction towards aspect $_{j}$ of entity $_{i}$, denoted as

$y_{\text {entity }_{i}}^{\text {aspect }_{i}}$, is calculated as Equation (5) shows.

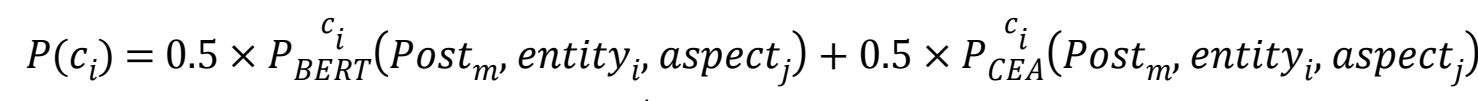

$$
\begin{aligned}
& y_{\text {entity }_{i}}^{\text {aspect }_{j}}=\operatorname{argmax} P\left(c_{i}\right)
\end{aligned}
$$

where $c_{i} \in\{$ positive, neutral, negative $\}, P\left(c_{i}\right)$ represents the probability that the sentiment is $c_{i}, P_{\text {model }} \stackrel{{ }^{c_{i}}}{c_{\text {most }}}$, entity $_{i}$, aspect $\left._{j}\right)$ represents the predicted probability of the sentiment $c_{i}$ towards aspect $_{j}$ of entity $_{i}$ in Post $_{m}$ by the basic model BERT or data augmented CEA.

\section{Time Complexity Analysis}

Compared with existing deep learning-based models, our proposed PEA model involves entity replacement, dual noise injection and prediction fusion as additional modules. The analysis of time complexity for these three parts is described as follows.

For entity replacement, we calculated the selected probability for every entity, whose time complexity is $O(E)$, where $E$ is the total number of entities in the dataset. We then traversed every instance and conduct entity replacement, whose time complexity is $O(N)$, where $N$ is the number of instances in the data set. The total time complexity of entity replacement is $O(E)$ $+O(N)$

For dual noise injection, we traversed every token in each instance to find the tokens referring to entity and aspect, whose time complexity is $O(T)$, where $T$ is the length of each instance. We 
385

386

387

388

389

390

391

392

393

394

395

396

397

398

399

400

401

402

403

404

405

406

407

408

409

410

411

412

413

414

415

416

417

418

419

420

421

added dual noises on all instances, whose time complexity is also $O(N)$. The total time complexity of dual noise injection is $O(T) \times O(N)$.

For prediction fusion, we fused the prediction with the weighted summation operation on every category for each instance, whose time complexity is $O(c) \times O(N)$, where $c$ is the number of categories of sentiments.

The total time complexity of extra operations in our proposed PEA model is $(O(E)+O(N))$ $+(O(T) \times O(N))+(O(c) \times O(N))$.

\section{Experiments and Analysis}

In this section, we introduce the experimental settings and results to validate the effectiveness of our PEA model.

\section{Experimental Settings}

We evaluate four benchmark datasets of three tasks, including datasets in two languages: English and Chinese. Statistics of the used datasets are displayed in Table 3.

- Restaurant and Laptop are two datasets from SemEval 2014 (Pontiki et al., 2014) for ABSA. Both datasets are reviews in English and each review contains aspects and corresponding sentiment polarities, including positive, negative and neutral.

- SentiHood is a widely used dataset for TABSA (Saeidi et al., 2016). It consists of 5,215 sentences in English, and 3,862 of which contain a single aspect, the rest contains multiple aspects. Each sentence is annotated with a list of tuples, which are aspect, given entity and corresponding sentiment polarity, including positive and negative. The whole dataset is split into train, validation and test set.

- BabyCare is a large public dataset for MEABSA (Yang et al. 2018). It consists of babycare reviews in Chinese and each review is in the format of a list of tuples, which are context, aspects, corresponding entities and sentiment polarities, including positive, negative and neutral. The whole dataset is split into train, validation and test set.

Common settings. For the BERT and CEA models, we use default parameters. For all English datasets, we use BERT-Base English models ${ }^{1}$ and $6 \mathrm{~B} 300 \mathrm{~d}$ GloVe (Pennington, Socher \& Manning, 2014) word embeddings ${ }^{2}$. For the Chinese dataset, we use BERT-Base Chinese and the same word vectors provided by (Yang et al., 2018). For multi-word entity terms and aspect terms, we follow the preprocessing in previous works (Yang et al., 2018; Song et al., 2019; Yang et al., 2019). We use the average vectors of all the words in the entity/aspect term as the entity/aspect term vectors.

Task specific settings. For ABSA task, the Restaurant and Laptop datasets are used for experiments. Because there is no entity in these datasets, so entity replacement in data augmentation is removed when implementing PEA. For TABSA task, the SentiHood dataset is used for experiments. Because aspect location is not given in this dataset, aspect noise injection

\footnotetext{
${ }^{1}$ https://github.com/google-research/bert.
}

${ }^{2}$ https://nlp.stanford.edu/projects/glove/ 
422 is removed in this task. For MEABSA task, the BabyCare dataset is used for experiments. When

423 implementing PEA, both entity replacement and noise injection are remained in this task.

424 Data augmentation settings. We perform entity replacement on the training data for the whole

425 dataset and merge the pseudo instances with original instances. According to the proposed entity

426 replacement method, those entities, which are low-resource in the original training set, have a

427 higher probability to be chosen for replacement. Table 4 lists the top 10 low-resource entities in

428 the BabyCare dataset, and displays the number of instances that belong to every category for

429 both the original training set and the entity-replaced dataset. We can observe that, for those low-

430 resource entities, such as "Kabrita", the number of negative and neutral instances has

431 significantly increased by using entity replacement. This can help relieve both the low-resource

432 and polarity bias problems.

433 For noise injection, $\mu$ and $\sigma$ are two parameters to be determined. We follow the common setting

434 in previous works (Kim, Roh \& $\operatorname{Kim}, 2019$ ) for $\mu$, which is $\mu=0$. For $\sigma$, we conduct

435 experiments on all four datasets with $\sigma$ ranging from 0.01 to 0.4 to quantify the noise level.

436 Experimental results are in Fig. 4.

437 The $x$-axis refers to different values of $\sigma$, the $y$-axis refers to the Macro-F1 performance. Four

438 lines with different kinds of marks refer to the results of four datasets. Experimental results show

439 that when $\mu=0$ and $\sigma=0.05$, noise injection achieves the utmost performance on all tasks. We

440 use this setting in the following experiments.

441 Model implementation settings. We implement our proposed model with TensorFlow 2.1,

442 Python 3.7. The device we used consists of CPU (E5 $2630 \mathrm{v} 4$ ), GPU (1080ti * 4) and RAM

443 (256G). We compare our model with the state-of-the-art baselines on 3 tasks predicting

444 sentiment towards entities and aspects.

\section{Results}

446 Accuracy and Marco-F1 score are two main-stream metrics in most sentiment analysis research,

447 where Marco-Fl is the $F 1$ score averaged over all the classes. In the following experiments,

448 Marco-Precision, Macro-Recall and AUC score are also used according to different tasks.

449 Results on the ABSA Task. We evaluate the English benchmark datasets ${ }^{3}$ Restaurant and

450 Laptop for the ABSA task. We compare with the published state-of-the-art baselines, including

451 Target-Dependent Long Short-Term Memory (TD-LSTM) (Tang et al., 2016), MemNet (Tang,

452 Qin \& Liu, 2016), Attention-based LSTM with Aspect Embedding (ATAE-LSTM) (Wang et al.,

453 2016), Interactive Attention Network (IAN) (Ma et al., 2017), Recurrent Attention on Memory

454 (RAM) (Chen et al., 2017), Transfer Capsule Network (TransCap) (Chen \& Qian, 2019), Aspect-

455 specific Graph Convolutional Network (ASGCN) (Zhang, Li \& Song, 2019), and Capsule

456 Network with Interactive Attention (IACapsNet) (Du et al., 2019). Following the former

457 research, Accuracy and Marco-F1 are evaluated for both datasets, Marco-Precision and Macro-

458 Recall are also reported. There is no entity in the dataset, so entity replacement in data

459 augmentation is removed. Results on two ABSA datasets are shown in Table 5.

460 We can have the following observations:

${ }^{3}$ http://alt.qcri.org/semeval2014/task4/ 
461 (1) by observing the accuracy and F1 performance, two Capsule Network-based models

462 TransCap and IACapsNet are much better than other previous baselines. This is because the key 463 components of TransCap and IACapsNet are recurrent neural works and attention mechanisms.

464 It shows that the RNN-based model has advantages in predicting fine-grained sentiments over 465 conventional methods.

466 (2) by observing the precision and recall on both datasets, the recall scores of most models 467 include TD-LSTM, ATAE-LSTM, IAN, RAM and ASGCN are much worse, while PEA can 468 have better performance.

469 (3) compared with all the baselines, our proposed model PEA achieves significant improvements 470 on both datasets. The experimental results show the PEA model is superior to other baselines in 471 the ABSA task under all evaluation metrics.

472 Results on the TABSA Task. We evaluate the English benchmark dataset SentiHood for the 473 TABSA task. It consists of 5,215 sentences, 3,862 of them contain a single target, and the 474 remainder multiple targets. We compare with all the published state-of-the-art baselines, 475 including Logistic Regression (LR) (Saeidi et al., 2016), LSTM+TA+SA (Ma, Peng \& Cambria, 476 2018), SenticLSTM (Ma, Peng \& Cambria, 2018), Dmu-Entnet (Liu, Cohn \& Baldwin, 2018), 477 RE+Delayed-memory (Liang et al., 2019), BERT-pair-QA-B and BERT-pair-QA-M (Sun, 478 Huang \& Qiu, 2019). Following the former research in the TABSA task, Accuracy and AUC are 479 usually reported and used as evaluation metrics, in the paper, Marco-Precision, Macro-Recall 480 and Marco-F1 are also reported. Results on TABSA are presented in Table 6.

481 We can have the following observations:

482 (1) BERT-pair-QA-M and BERT-pair-QA-B are the previous state-of-the-art models. Compared 483 with other none-BERT based baselines, BERT-pair-QA-M and BERT-pair-QA-B outperform the 484 LR, LSTM+TA+SA, SenticLSTM, Dmu-Entnet and RE+Delayed-memory models in both 485 accuracy and AUC score. This result shows the effectiveness of the pre-trained language model 486 for fine-grained sentiment analysis.

487 (2) compared with two BERT-based baselines, our proposed PEA achieves further improvement 488 in most evaluation metrics. This may be because the prediction of PEA comes from both data 489 augmented CEA and BERT, which helps ensemble the predictions of two basic models.

490 (3) different from the performance in ABSA and MEABSA, the improvement of PEA in the 491 TABSA task seems slightly in accuracy and AUC score, this may be because aspect location is 492 not given in this dataset (but given in other tasks), therefore, aspect noise injection is removed 493 for this experiment. So we have conducted a statistical analysis test in the following section to 494 show the performance difference between the two models is statistically significant.

495 Results on the MEABSA Task. We evaluate the Chinese benchmark dataset BabyCare for the 496 MEABSA task. We compare with all the published state-of-the-art baselines, including CEA 497 (Yang et al., 2018), DT-CEA (Yang et al., 2019), Cold-start Aware Deep Memory Network 498 (CADMN) (Song et al., 2019). These methods are exactly designed for this task. We also 499 compare with MemNet (Tang, Qin \& Liu, 2016), ATAE-LSTM (Wang et al., 2016), IAN (Ma et 500 al., 2017), and their modified versions MemNet+, ATAE-LSTM+ and IAN+, which are used as 
501 baselines in a recent MEABSA work (Song et al., 2019). We follow the designs introduced in 502 (Song et al., 2019): these three modified plus versions remain the basic model structure of 503 MemNet, ATAE-LSTM and IAN respectively. The additional entities in the MEABSA task are 504 treated as the aspects, and are added to the models in the same manner of aspects. These methods 505 are originally designed for the ABSA task, and they are often regarded as baselines in former 506 MEABSA research. Following the former research, Accuracy and Marco-F1 are evaluation 507 metrics for this dataset, Marco-Precision and Macro-Recall are also reported. Table 7 displays 508 the comparisons between our model and baselines.

509 We can have the following observations:

510 (1) MemNet, ATAE-LSTM, and IAN in the first three lines only model aspects while ignoring 511 entity modeling. Their performances are worse than the plus versions MemNet+, ATAE-

512 LSTM+, and IAN+, which model the entity in the same manner as aspect, illustrating the 513 effectiveness of entity modeling in the MEABSA task.

514 (2) The CEA model combines the advantages of both attention-based LSTM and deep memory 515 networks, the former is the key component of ATAE-LSTM+ and the latter is the key component 516 of MemNet+. The performance of CEA is much better than ATAE-LSTM+ and MemNet+, 517 which reaches about $15 \%$ in accuracy. This shows that the CEA model has advantages in the 518 MEABSA task, and is more suitable to be chosen as an RNN-based basic model for PEA.

519 (3) DT-CEA and CADMN are two extension models based on CEA. DT-CEA incorporated 520 dependency information to improve CEA. CADMN used a frequency-guided attention 521 mechanism to improve CEA. The performance of CADMN and DT-CEA are comparable to each 522 other and are little better than CEA.

523 (4) compared with all the baselines, our proposed method PEA achieves significant improvement

524

525

526

527

528

529

530

531

532

533

534

535

536

537

538

539

540 under all evaluation metrics. Compared with the previous state-of-the-art CADMN model, the improvements of PEA reach about $4 \%$ in accuracy and $5 \%$ in F1. The MEABSA is the most challenging fine-grained sentiment analysis task, this experimental result shows PEA has a significant advantage in the MEABSA task.

Statistical Analysis Test. Refer to the previous works (Li et al., 2020), we conduct McNemars test as the statistical analysis test to further show the statistical difference between two models. $p$ -value is the significance level, which means the performance difference between the two models. If the estimated $p$-value is lower than 0.05 , the performance difference between the two models is statistically significant. Table 8 displays the $p$-values between PEA and other models on three sentiment analysis tasks respectively.

We can observe that the performance differences between PEA and other baselines are statistically significant in all tasks, which show the effectiveness of the proposed PEA model from the perspective of statistical analysis. For example, in the TABSA task, the improvement of PEA compared with BERT-pair-NLI-M is not very high in accuracy, which is $94.3 \%$ vs $93.8 \%$ in Table 6. In the statistical analysis test, the estimated $p$-value between PEA and BERT-pairNLI-M is 0.0174 . According to the definition of $p$-value, it shows that the performance difference between BERT-pair-NLI-M and PEA is statistically significant. Additionally, by

Peer) Comput. Sci. reviewing PDF | (CS-2021:10:66426:1:0:NEW 14 Nov 2021) 
541 observing Table 7 and Table 8 together, we can find PEA has significant advantages in the most 542 challenging MEABSA task.

543

544 Ablation Study

545 Experimental results so far show that the PEA approach is superior to the baselines on all the

546 ABSA, TABSA and MEABSA on selected datasets. Because PEA consists of data augmented

547 CEA and BERT, we would like to further investigate the effectiveness of each part in the model.

548 A case study is also introduced in this section.

549 Effectiveness of Components in PEA

550 Ablation study is used to show how each part of the model affects the performance by removing

551 them. We conduct experiments on all four datasets of three tasks for comparisons. Experimental

552 results are as Table 9 shows.

553 The proposed PEA model integrates data augmented CEA and BERT. Because entity

554 replacement and noise injection are applied to data augmented CEA, we use CEA,

555 CEA+EntityReplacement (CEA+ER for short) and CEA+EntityReplacement+NoiseInjection

556 (CEA+ER+NI for short) respectively for ablation study to show the effectiveness of applying

557 two data augmentation techniques. The BERT-based model is also used for comparisons in

558 ablation studies.

559 We can have the following observations from Table 9:

560 (1) comparing CEA and CEA+ER, we can find involving entity replacement can have

561 improvement on MEABSA and TABSA tasks. We also counted the number of instances for

562 every entity based on the original training set and the entity-replaced dataset. The statistics are

563 demonstrated with the box plot in Fig. 5.

564 It shows that using the proposed entity-replacement method can significantly increase the

565 number of instances for low-resource entities, and all entities have at least 252 instances for

566 training. For ABSA, there is no entity provided in the dataset, so the entity replacement

567 procedure is removed.

568 (2) by adding noise injection, the CEA+ER+NI model achieves about $1.3 \%$ improvement on the

569 Restaurant dataset over the CEA+ER model, and achieves slight improvement on other datasets.

570 These observations show that using entity replacement and noise injection can bring positive

571 impacts on fine-grained sentiment analysis. This may be because using data augmentation can

572 increase the number of training instances, especially for low-resource entities and aspects, and

573 help overcome polarity bias.

574 (3) by comparing the performance of PEA with the BERT-based model and data augmented

575 CEA model, PEA achieves the best performance in most cases. The strength of BERT-based

576 model is that it makes use of a huge amount of unlabeled data by pre-training, but it also has

577 weaknesses. The BERT model depends on the Transformer (Vaswani et al., 2017), which further

578 mainly relies on its self-attention mechanism. It has been suggested that self-attention has

579 limitations that it cannot process input sequentially (Dehghani et al., 2018; Hao et al., 2019; Shen

580 et al., 2018; Hahn, 2020). Such a weakness is just the strength of recurrent neural networks, 
581 which is one of the core components in CEA. Our model PEA combines the advantages of both

582

583

584

585

586

587

588

589

590

591

592

593

594

595

596

597

598

599

600

601

602

603

604

605

606

607

608

609

610

611

612

613

614

615

616

617 The $x$-axis refers to the percentage of data used for training, the $y$-axis refers to the Macro-F1 of

618 different models. ER and NI are the abbreviations of entity replacement and noise injection. We

619 and performs the best in most cases. To better understand the strengths and weaknesses of data augmented CEA and BERT, we carry out a case study in the next section.

\section{Case Study}

We give empirical validation on the strengths and weaknesses of two basic models, including the BERT-based model and data augmented CEA, by a further case study on misclassifications of both models. We test on the most challenging task MEABSA and use the corresponding Babycare dataset for the case study. To show the stability of the models rather than the occasionality, we have trained the BERT-based model, the Data augmented CEA model and the PEA model five times. The predictions of two representative examples are as Table 10 shows. For example 1, the BERT-based model makes the same misclassification on the inputs five times and the data augmented CEA model achieves the correct predictions. Example 2 is just the opposite. Such stable misclassifications reveal the defects of both models. The first example has a special pattern: the coreference structure of "...the former..., ...the latter...". The second example consists of two simple sentences. Correctly predicting the first example need the ability of global sequence or structure understanding which is the advantage of recurrent neural networks. The recurrent neural network is one of the core components of CEA. Correctly predicting the second example need the ability of local attention which is the advantage of self-attention, which is the core component of the BERT-based model. PEA fuses the prediction with both BERT-based model and data augmented CEA model based on ensemble methods, which make the correct prediction on both examples. This case study further helps illustrate the value and necessity of ensembling two basic models.

We also give the third example in Table 10, where all the CEA, BERT-based model and PEA made the wrong prediction. The gold output should be negative, but all models predicted it as neutral. The possible reason is that there are no aspect terms directly towards the target entity 'Kao', which cause the model to give the prediction as neutral.

\section{Results on Challenging Conditions}

There are two challenges in sentiment prediction towards entities and aspects: the low-resource problem and the polarity bias problem. In this section, we evaluate the negative effect of challenges and the ability of models to solve them.

\section{Results on Extreme Low-Resource Conditions}

To further test the model's performance under extreme low-resource conditions, we randomly selected 5\%,10\%,20\%, and 50\%, each time, from the original dataset as our training dataset. All tests are performed under the most challenging Babycare dataset. Experimental results are as Fig. 6 shows. can have the following observations from Fig. 6. 
620 (1) for all models, as the percentage of the training set used decreases, the models' performance

621

622

623

624

625

626

627

628

629

630

631

632

633

634

635

636

637

638

639

640

641

642

643

644

645

646

647

648

649

650

651

652

653

654

655

656

657

658

drops significantly, which further illustrates the significance of the low-resource problem on sentiment prediction.

(2) CEA+ER outperforms the CEA model under all the low-resource conditions, which shows the effectiveness of using entity replacement. By using noise injection, the $\mathrm{CEA}+\mathrm{ER}+\mathrm{NI}$ achieves further improvements over CEA and CEA+ER.

(3) for the BERT-based model, when the resource is extremely low, the BERT-based model deteriorates sharply. For example, when $5 \%$ of data is used for training, the Macro-F1 of BERTbased model and PEA is $57.16 \%$ vs $64.37 \%$. This shows that the combination of the data augmented CEA and BERT-based model for PEA can boost the stability of the model. (4) the dotted line in red refers to the baseline results with $100 \%$ data for training, we can observe from Fig. 6 that when only 20\% data are used for training, the proposed PEA can achieve a similar performance of the CEA model with full-resource data for training. With the size of training data becoming larger, the improvement of PEA becomes more obvious. This shows the PEA model, which combines data augmented CEA with BERT-based model, has advantages under lowresource conditions.

\section{Results on Evident Polarity Biased Conditions}

Polarity bias occurs when sentiment polarity distribution towards an entity is not uniform.

Polarity bias reduces the performance when sentiments towards an entity diverge in the training set and in the test set (e.g. $70 \%$ of sentiment towards entity A are positive in the training set while $60 \%$ of which are negative in the test set). We create a new test set named EPB test set, which consists of all the instances with entities polarity biased from the original test set. Using the BabyCare test set, we find entities in $30 \%$ of data (1070 out of 3677 ) have the evident polarity bias problem. Experimental results are as Table 11 shows, the last column displays the decline between the performance on the Original test set and EPB test set.

After comparing the sentiment prediction results from using the evident polarity biased data with the results from using the origin data, we have the following observations:

(1) the performance of all models has varying degrees of decline on the polarity biased EPB dataset. This shows the polarity bias problem is one of the challenges in fine-grained sentiment analysis.

(2) comparing CEA and CEA+DA, the performance on the EPB test dataset is close to the performance on the original test set. This is because data augmentations can relieve the polarity bias problem by providing plenty, omni-polar sentiment training data, and reduced the variance of test results to offer more stable performance. This shows applying data augmentations can address the polarity bias problem in fine-grained sentiment analysis and make the model more generality.

(3) comparing CEA and the BERT-based model, the performance on the original test set of the BERT-based model has a significant improvement than that of CEA. (4) PEA achieves the best performance on the original test set, and relieves the polarity problem on the EPB test at the

Peer] Comput. Sci. reviewing PDF | (CS-2021:10:66426:1:0:NEW 14 Nov 2021) 
659 same time, which also shows the necessity and effectiveness of using the ensemble methods to

660

661

662

663

664

665

666

667

668

669

670

671

672

673

674

675

676

678

680

682

683

684

685

686

687

688

689

690

691

692

693

694

695

696

697

698

699

fuse the predictions of CEA and BERT based models with data augmentations.

\section{Conclusions}

In this paper, we developed the PEA model, which unified the ABSA, TABSA, and MEABSA tasks together for the first time and provided an all-in-one solution to interpret consumers' opinions on all kinds of social media platforms. For the first time, we analysed the effect of the sentiment polarity bias problem in these tasks. Most importantly, we created two innovative, task-specific methods to alleviate the low-resource problem and the polarity bias problem, not only getting promising experimental results, but also providing inspiration for successors to make more contributions in this area. For future work, there are two possible extensions worth considering. The first one is to look for new ways to combine pre-trained language models with RNN-based models, to integrate both advantages. The second one is to further investigate more types of fine-grained sentiment analysis, and propose unified models handling various finegrained sentiment-related tasks, for example, emotion cause analysis.

\section{Acknowledgements}

We would like to sincerely thank our from Jiangnan University, Professor JunUniversity, for their kind support and

colleagues, Professor Xiao-jun Wu, and Jun Sun yuan Xie, and Chong-jun Wang from Nanjing guidance.

\section{References}

Acheampong F, Nunoo-Mensah H, Chen W. Transformer models for text-based emotion detection: a review of BERT-based approaches. Artificial Intelligence Review, 2021: 1-41.

Chen P, Sun Z, Bing L, Yang W. Recurrent attention network on memory for aspect sentiment analysis. in Proceedings of EMNLP, Copenhagen, Denmark, 2017: 452-461.

Chen Z, Qian T. Transfer capsule network for aspect level sentiment classification. in Proceedings of ACL, Florence, Italy, 2019: 547-556.

Chen Z, Qian T. Relation-aware collaborative learning for unified aspect-based sentiment analysis. in Proceedings of ACL, Seattle, Washington, USA, 2020: 3685-3694.

Cheng Y, Tu Z, Meng F, Zhai J, Liu Y. Towards Robust Neural Machine Translation. in Proceedings of ACL, Melbourne, Australia, 2018: 1756-1766.

Dai J, Yan H, Sun T, Liu P, Qiu X. Does syntax matter? A strong baseline for Aspect-based Sentiment Analysis with RoBERTa. in Proceedings of NAACL-HLT, online, 2021: 1816-1829.

Dai X, Adel H. An Analysis of Simple Data Augmentation for Named Entity Recognition. in Proceedings of COLING, Barcelona, Spain, 2020: 3861-3867.

Dehghani M, Gouws S, Vinyals O, Uszkoreit J, Kaiser $\mathbf{L}$. Universal Transformers. in Proceedings of ICLR, Vancouver, BC, Canada. 2018. 
700 Devlin J, Chang M.W, Lee K, Toutanova K. Bert: Pre-training of deep bidirectional 701 transformers for language understanding. in Proceedings of NAACL-HTL, New Orleans, 702 Louisiana, USA, 2018: 4171-4186.

703 Du C, Sun H, Wang J, Qi Q, Liao J, Xu T, Liu M. Capsule network with interactive attention 704 for aspect-level sentiment classification. in Proceedings of EMNLP-IJCNLP, Hong Kong, China, 705 2019: 5492-5501.

706 Fadaee M, Bisazza A, Monz C. Data Augmentation for Low-Resource Neural Machine 707 Translation. in Proceedings of ACL, Vancouver, Canada, 2017: 567-573.

708 Gupta R. Data augmentation for low resource sentiment analysis using generative adversarial 709 networks. in Proceedings of ICASSP, Brighton, UK, 2019: 7380-7384.

710 Hahn M. Theoretical limitations of self-attention in neural sequence models. Transactions of the 711 Association for Computational Linguistics, 2020, 8: 156-171.

712 Han H, Zhang Y, Zhang J, Yang J, Zou X. Improving the performance of lexicon-based 713 review sentiment analysis method by reducing additional introduced sentiment bias. PloS one, 714 2018, 13(8): e0202523.

715 Hao J, Wang X, Yang B, Wang L, Zhang J. Modeling Recurrence for Transformer. in 716 Proceedings of NAACL-HTL, Minneapolis, MN, USA, 2019: 1198-1207.

717 Hong H, Song J. Utilizing BERT for Detecting Aspect Categories on TABSA via Adjusting 718 Self-attention among Words. in Proceedings of ICHCI, Sanya, China, 2020: 66-70.

719 Hou Y, Liu Y, Che W, Liu T. Sequence-to-Sequence Data Augmentation for Dialogue

720 Language Understanding. in Proceedings of COLING, Santa Fe, New Mexico, USA, 2018 :

721 1234-1245.

722 Howard J, Ruder S. Universal Language Model Fine-tuning for Text Classification. in 723 Proceedings of ACL, Melbourne, Australia, 2018: 328-339.

724 Iqbal M, Karim A, Kamiran F. Bias-aware lexicon-based sentiment analysis. in Proceedings of 725 SAC, Salamanca, Spain, 2015: 845-850.

726 Khine W, Aung T. Applying deep learning approach to targeted aspect-based sentiment analysis 727 for restaurant domain. in Proceedings of ICAIT, Jinan, China, 2019: 206-211.

728 Kim H.Y, Roh Y.H, Kim Y.G. Data augmentation by data noising for open-vocabulary slots in 729 spoken language understanding. in Proceedings of NAACL-HTL, Minneapolis, MN, USA, 2019: $730 \quad 97-102$.

731 Kiritchenko S, Mohammad S. Examining Gender and Race Bias in Two Hundred Sentiment 732 Analysis Systems. in Proceedings of SIGLEX-SIGSEM, New Orleans, Louisiana, USA, 2018: 733 43-53.

734 Li Z, Sun J, Wu X, Yin H. Multiplication fusion of sparse and collaborative-competitive 735 representation for image classification. International Journal of Machine Learning and 736 Cybernetics, 2020, 11(10): 2357-2369.

737 Liang B, Du J, Xu R, Li B, Huang H. Context-aware Embedding for Targeted Aspect-based 738 Sentiment Analysis. in Proceedings of ACL, Florence, Italy, 2019: 4678-4683. 
739 Liu F, Cohn T, Baldwin T. Recurrent Entity Networks with Delayed Memory Update for

740 Targeted Aspect-Based Sentiment Analysis. in Proceedings of NAACL, New Orleans,

741 Louisiana, USA. 2018: 278-283.

742 Lu K, Mardziel P, Wu F, Amancharla P, Datta A. Gender bias in neural natural language

743 processing. Logic, Language, and Security, 2020: 189-202.

744 Ma D, Li S, Zhang X, Wang H. Interactive attention networks for aspect-level sentiment

745 classification. in Proceedings of IJCAI, Melbourne, VIC, Australia, 2017: 4068-4074.

746 Ma Y, Peng H, Cambria E. Targeted aspect-based sentiment analysis via embedding

747 commonsense knowledge into an attentive LSTM. in Proceedings of AAAI, New Orleans, LA,

748 USA, 2018: 5876-5883.

749 Peters M.E, Neumann M, Iyyer M, Gardner M, Clark C, Lee K, Zettlemoyer L. Deep

750 Contextualized Word Representations. in Proceedings of ACL, Melbourne, Australia, 2018:

$751 \quad 2227-2237$.

752 Pennington J, Socher R, Manning C. D. Glove: Global vectors for word representation. in

753 Proceedings of EMNLP, Doha, Qatar, 2014: 1532-1543.

754 Pontiki M, Papageorgiou H, Galanis D, Androutsopoulos I, Pavlopoulos J, Manandhar S.

755 SemEval-2014 Task 4: Aspect Based Sentiment Analysis, in Proceedings of SemEval, Dublin,

756 Ireland, 2014: 27-35.

757 Saeidi M, Bouchard G, Liakata M, Riedel S. SentiHood: Targeted Aspect Based Sentiment

758 Analysis Dataset for Urban Neighbourhoods. in Proceedings of COLING, Osaka, Japan, 2016:

759 1546-1556.

760 Sagi O, Rokach L. Ensemble learning: A survey. Wiley Interdisciplinary Reviews: Data Mining

761 and Knowledge Discovery, 2018, 8(4): e1249.

762 Song K, Gao W, Zhao L, Sun C, Liu X. Cold-start aware deep memory networks for multi-

763 entity aspect-based sentiment analysis. in Proceedings of IJCAI, Macao, SAR, China, 2019:

764 5197-5203.

765 Shen T, Zhou T, Long G, Jiang J, Pan S, Zhang C. Disan: Directional self-attention network

766 for $\mathrm{rnn} / \mathrm{cnn}$-free language understanding. in Proceedings of AAAI, New Orleans, LA, USA,

767 2018: 5446-5455.

768 Sun C, Huang L, Qiu X. Utilizing BERT for Aspect-Based Sentiment Analysis via

769 Constructing Auxiliary Sentence. in Proceedings of ACL, Florence, Italy, 2019: 380-385.

770 Tang D, Qin B, Feng X, Liu T. Effective LSTMs for Target-Dependent Sentiment

771 Classification. in Proceedings of COLING, Osaka, Japan, 2016: 3298-3307.

772 Tang D, Qin B, Liu T. Aspect Level Sentiment Classification with Deep Memory Network. in

773 Proceedings of EMNLP, Austin, Texas, USA, 2016: 214-224.

774 Thelwall M. Gender bias in machine learning for sentiment analysis. Online Information

775 Review, 2018, 42(1): 45-57.

776 Vaswani A, Shazeer N, Parmar N, Uszkoreit J, Jones L, Gomez A.N, Polosukhin I.

777 Attention is all you need. in Proceedings of NeurIPS, Long Beach, CA, USA, 2017: 6000-6010. 
778

779

780

781

782

783

784

785

786

787

788

789

790

791

792

793

794

795

796

797

798

799

800

801

802

803

804

805

806

807

808

809

810

811

812

813

814

van Aken B, Winter B, Löser A, Gers F. How does bert answer questions? a layer-wise analysis of transformer representations. In Proceedings of CIKM, Beijing, China, 2019: 18231832.

Wan H, Yang Y, Du J, Liu Y, Qi K, Pan J. Target-aspect-sentiment joint detection for aspectbased sentiment analysis. in Proceedings of AAAI, New York, New York, USA, 2020: 91229129.

Wang K, Shen W, Yang Y, Quan X, Wang R. Relational Graph Attention Network for Aspectbased Sentiment Analysis. in Proceedings of ACL, Online Conference, 2020: 3229-3238. Wang W.Y, Yang D. That's so annoying!!!: A lexical and frame-semantic embedding based data augmentation approach to automatic categorization of annoying behaviors using\# petpeeve tweets. in Proceedings of EMNLP, Lisbon, Portugal, 2015: 2557-2563.

Wang Y, Huang M, Zhu X, Zhao L. Attention-based LSTM for aspect-level sentiment classification. in Proceedings of EMNLP, Austin, Texas, USA, 2016: 606-615.

Wu Z Ong D. Context-guided bert for targeted aspect-based sentiment analysis. in Proceedings of AAAI, New York, New York, USA, 2020: 1-9.Xia M, Kong X, Anastasopoulos A, Neubig G. Generalized Data Augmentation for Low-Resource Translation. in Proceedings of ACL, Florence, Italy, 2019: 5786-5796.

Xu B, Wang X, Yang B, Kang Z. Target embedding and position attention with 1stm for aspect based sentiment analysis. in Proceedings of ICMAI, Chengdu, China, 2020: 93-97.

Yang J, Yang R, Wang C, Xie J. Multi-entity aspect-based sentiment analysis with context, entity and aspect memory. in Proceedings of AAAI, New Orleans, LA, USA, 2018: 6029-6036. Yang J, Yang R, Lu H, Wang C, Xie J. Multi-entity aspect-based sentiment analysis with context, entity, aspect memory and dependency information. ACM TALLIP, 2019, 18(4): 1-22. Ye Z, Li Z. A variant of recurrent entity networks for targeted aspect-based sentiment analysis. in Proceedings of ECAI, Santiago de Compostela, Spain, 2020: 2268-2274.

Zhao J, Wang T, Yatskar M, Ordonez V, Chang K. W. Gender Bias in Coreference Resolution: Evaluation and Debiasing Methods. in Proceedings of NAACL, New Orleans, Louisiana, USA, 2018: 15-20.

Zhang C, Li Q, Song D. Aspect-based Sentiment Classification with Aspect-specific Graph Convolutional Networks. in Proceedings of EMNLP-IJCNLP, Hong Kong, China, 2019: 45604570 .

Zhang M, Qian T. Convolution over Hierarchical Syntactic and Lexical Graphs for Aspect Level Sentiment Analysis. in Proceedings of EMNLP, online, 2020: 3540-3549.

Zhou Z. Ensemble learning. Machine Learning. Springer, Singapore, 2021: 181-210.

Zhu P, Chen Z, Zheng H, Qian T. Aspect aware learning for aspect category sentiment analysis. ACM Transactions on Knowledge Discovery from Data. ACM TKDD, 2019, 13(6): 121. 


\section{Table $\mathbf{1}$ (on next page)}

Table 1. The comparison between three tasks of sentiment prediction towards entities and aspects. 
Table 1. The comparison between three tasks of sentiment prediction towards entities and aspects.

\begin{tabular}{|c|c|c|c|c|}
\hline & & ABSA & TABSA & MEABSA \\
\hline \multirow[b]{2}{*}{ Definition } & Given & text and aspects mentioned & $\begin{array}{l}\text { text, entity mentioned, and all kinds } \\
\text { of aspects }\end{array}$ & $\begin{array}{l}\text { text, entity mentioned, and aspect } \\
\text { mentioned }\end{array}$ \\
\hline & Goal & $\begin{array}{l}\text { predict sentiment towards } \\
\text { mentioned aspects }\end{array}$ & $\begin{array}{l}\text { predict sentiment towards the } \\
\text { combination of mentioned entity } \\
\text { and all kinds of aspects }\end{array}$ & $\begin{array}{l}\text { predict sentiment towards mentioned } \\
\text { entity aspect combination }\end{array}$ \\
\hline \multirow[t]{2}{*}{ Example1 } & Input & $\begin{array}{l}<\text { context }>\text { I've used } \\
\text { MacBookPro, it's } \\
\text { convenient. }<\text { /context }> \\
<\text { aspect from =" } 27 " \\
\text { to="“37"> convenience } \\
\text { level }</ \text { aspect }>\end{array}$ & $\begin{array}{l}<\text { context }>\text { I've used MacBookPro, } \\
\text { it's convenient. }</ \text { context }><\text { entity } \\
\text { from="10" to=" } 20 ">\text { MacBookPro } \\
<\text { entity }><\text { aspectlist }>\text { price, } \\
\text { convenience level, battery, ... } \\
</ \text { aspectlist }>\end{array}$ & $\begin{array}{l}<\text { context }>\text { I've used MacBookPro, it's } \\
\text { convenient. }</ \text { context }><\text { entity } \\
\text { from=" } 10 " \text { to=" } 20 ">\text { MacBookPro } \\
</ \text { entity }><\text { aspect }>\text { from=" } 27 " \\
\text { to=" } 37 \text { " }>\text { convenience level }</ \text { aspect }>\end{array}$ \\
\hline & Output & $\begin{array}{l}\text { (convenience level, } \\
\text { positive) }\end{array}$ & $\begin{array}{l}\text { (MacBookPro, price, none) } \\
\text { (MacBookPro, convenience level, } \\
\text { positive) (MacBookPro, battery, } \\
\text { none) ... }\end{array}$ & $\begin{array}{l}\text { (MacBookPro, convenience level, } \\
\text { positive) }\end{array}$ \\
\hline \multirow[t]{2}{*}{ Example2 } & Input & $\begin{array}{l}<\text { context }>\text { The battery of } \\
\text { ThinkPad is very long. } \\
</ \text { context }><\text { aspect from } \\
=\text { " } 4 \text { " to=" } 11 \text { " }> \\
\text { battery }</ \text { aspect }>\end{array}$ & $\begin{array}{l}<\text { context }>\text { The battery of ThinkPad } \\
\text { is very long. }</ \text { context }><\text { entity } \\
\text { from=" } 15 " \text { to =" } 23 \text { " }>\text { ThinkPad } \\
<\text { entity }><\text { aspectlist }>\text { price, } \\
\text { convenience level, battery, ... } \\
<\text { /aspectlist }>\end{array}$ & $\begin{array}{l}<\text { context }>\text { The battery of ThinkPad is } \\
\text { very long. }</ \text { context }><\text { entity } \\
\text { from=" } 15 " \text { to=" } 23 \text { " }>\text { ThinkPad } \\
</ \text { entity }><\text { aspect }>\text { from=" } 4 " \\
\text { to=" } 11 ">\text { battery }</ \text { aspect }>\end{array}$ \\
\hline & Output & (battry, positive) & $\begin{array}{l}\text { (ThinkPad, price, none) (ThinkPad, } \\
\text { convenience level, none) } \\
\text { (ThinkPad, battery, positive) ... }\end{array}$ & (ThinkPad, battery, positive) \\
\hline
\end{tabular}

2 


\section{Table 2 (on next page)}

Table 2. An example of entity replacement. The replacement maintains the same sentiment polarity and correct grammar. 
1 Table 2. An example of entity replacement. The replacement maintains the same sentiment polarity and correct 2

\begin{tabular}{|c|c|l|}
\hline \multirow{2}{*}{ Original } & post & I've used MacBookPro, it's convenient. \\
\cline { 2 - 3 } & entity & MacBookPro \\
\hline \multirow{2}{*}{ New } & post & I've used Thinkpad, it's convenient. \\
\cline { 2 - 3 } & target entity & Thinkpad \\
\hline
\end{tabular}

3 


\section{Table 3(on next page)}

Table 3. Statistics of used datasets. 
1

Table 3. Statistics of used datasets.

\begin{tabular}{|c|c|c|c|c|c|c|c|}
\hline \multirow[b]{2}{*}{ Dataset } & \multirow[b]{2}{*}{ Language } & \multirow{2}{*}{$\begin{array}{c}\text { Trainin } \\
\text { g } \\
\text { set }\end{array}$} & \multirow{2}{*}{$\begin{array}{c}\text { Validatio } \\
\text { n set }\end{array}$} & \multirow{2}{*}{$\begin{array}{c}\text { Test } \\
\text { set }\end{array}$} & \multicolumn{3}{|c|}{ Task } \\
\hline & & & & & ABSA & $\begin{array}{c}\text { TABS } \\
\text { A }\end{array}$ & MEABSA \\
\hline Restaurant & English & 3,608 & - & 1,120 & B & & \\
\hline Laptop & English & 2,328 & - & 638 & $B$ & & \\
\hline SentiHood & English & 3,650 & 522 & 1,043 & & $B$ & \\
\hline BabyCare & Chinese & 29,354 & 3,682 & 3,677 & & & $B$ \\
\hline
\end{tabular}

2 


\section{Table 4(on next page)}

Table 4: Definition of the confusion matrix. 
Table 4. Top 10 low-resource entities in the BabyCare dataset, with the number of instances that belong to every polarity category for both the original training set and entity-replaced dataset.

\begin{tabular}{|l|l|l|l|l|l|l|}
\hline & \multicolumn{3}{|c|}{ the original training set } & \multicolumn{3}{c|}{ the entity-replaced dataset } \\
\hline \multicolumn{1}{|c|}{ Entity } & Negative & Neutral & Positive & Negative & Neutral & Positive \\
\hline 佳贝艾特(Kabrita) & 0 & 0 & 26 & 69 & 243 & 361 \\
\hline 可瑞康(Karicare) & 0 & 0 & 17 & 108 & 382 & 527 \\
\hline 君乐宝(JunLeBao) & 0 & 0 & 15 & 121 & 409 & 571 \\
\hline 咔哇熊(Cowala) & 0 & 0 & 14 & 144 & 446 & 642 \\
\hline 多美滋(Dumex) & 1 & 0 & 64 & 22 & 84 & 219 \\
\hline 太子乐(Happy Prince) & 1 & 0 & 19 & 102 & 306 & 485 \\
\hline 奶粉(milk powder) & 0 & 0 & 19 & 102 & 304 & 479 \\
\hline 欧贝嘉(OuBecca) & 1 & 0 & 19 & 86 & 305 & 459 \\
\hline 百立乐(Natrapure) & 4 & 0 & 73 & 37 & 71 & 183 \\
\hline 诺优能(Nutrilon) & 2 & 0 & 42 & 44 & 146 & 227 \\
\hline
\end{tabular}




\section{Table 5 (on next page)}

Table 5. Performance (\%) on two datasets for the ABSA task, Accuracy, Marco-Precision, Macro-Recall and Marco-Flare reported. 
1 Table 5. Performance (\%) on two datasets for the ABSA task, Accuracy, Marco-Precision, Macro-Recall and 2 Marco-Flare reported.

\begin{tabular}{|l|l|l|l|l|l|l|l|c|}
\hline \multirow{2}{*}{ Models } & \multicolumn{5}{|c|}{ Restaurant } & \multicolumn{5}{c|}{ Laptop } \\
\cline { 2 - 10 } & Accuracy & Precision & Recall & F1 & Accuracy & Precision & Recall & F1 \\
\hline TD-LSTM & 75.18 & 70.60 & 56.57 & 58.51 & 64.26 & 57.67 & 56.67 & 54.10 \\
\hline MemNet & 77.32 & 69.87 & 64.38 & 64.61 & 68.65 & 63.58 & 63.62 & 62.69 \\
\hline $\begin{array}{l}\text { ATAE- } \\
\text { LSTM }\end{array}$ & 74.38 & 67.43 & 57.28 & 58.32 & 66.14 & 61.22 & 58.97 & 56.91 \\
\hline IAN & 76.16 & 67.43 & 59.31 & 60.56 & 65.20 & 61.64 & 58.54 & 54.08 \\
\hline RAM & 76.07 & 72.07 & 58.65 & 59.59 & 68.03 & 64.03 & 63.86 & 60.82 \\
\hline TransCap & 79.20 & 70.76 & 70.81 & 70.78 & 74.76 & 71.77 & 71.99 & 70.08 \\
\hline ASGCN & 74.29 & 71.95 & 56.74 & 56.45 & 69.75 & 66.21 & 63.75 & 62.29 \\
\hline IACapsNet & 81.79 & - & - & 73.40 & 76.80 & - & - & 73.29 \\
\hline PEA(Our) & $\mathbf{8 4 . 8 2}$ & $\mathbf{8 0 . 4 1}$ & $\mathbf{7 6 . 3 1}$ & $\mathbf{7 8 . 1 4}$ & $\mathbf{7 8 . 6 8}$ & $\mathbf{7 4 . 4 3}$ & $\mathbf{7 6 . 6 0}$ & $\mathbf{7 5 . 0 7}$ \\
\hline
\end{tabular}




\section{Table 6(on next page)}

Table 6. Performance (\%) on the SentiHood dataset for the TABSA task, Accuracy, Marco-Precision, Macro-Recall, Marco-F1 and AUC are reported. 
1 Table 6. Performance (\%) on the SentiHood dataset for the TABSA task, Accuracy, Marco-Precision, Macro2 Recall, Marco-F1 and $A U C$ are reported.

\begin{tabular}{|l|l|l|l|l|l|}
\hline \multicolumn{1}{|c|}{ Models } & \multicolumn{1}{|c|}{ Accuracy } & Precision & \multicolumn{1}{|c|}{ Recall } & \multicolumn{1}{|c|}{ F1 } & \multicolumn{1}{c|}{ AUC } \\
\hline LR & 87.5 & - & - & - & 90.5 \\
\hline LSTM+TA+SA & 86.8 & - & - & - & - \\
\hline SenticLSTM & 89.3 & - & - & - & - \\
\hline Dmu-Entnet & 90.2 & 74.8 & 76.3 & 75.5 & 94.8 \\
\hline RE+Delayed-memory & 92.8 & - & - & - & 96.2 \\
\hline BERT-pair-QA-B & 93.3 & - & - & - & 97.0 \\
\hline BERT-pair-QA-M & 93.8 & 83.4 & $\mathbf{8 5 . 7}$ & 84.5 & 97.1 \\
\hline PEA(Our) & $\mathbf{9 4 . 3}$ & $\mathbf{8 6 . 0}$ & 84.5 & $\mathbf{8 5 . 2}$ & $\mathbf{9 7 . 4}$ \\
\hline
\end{tabular}




\section{Table 7 (on next page)}

Table 7. Performance (\%) on the BabyCare dataset for the MEABSA task, Accuracy, Marco-Precision, Macro-Recall and Marco-F1 are reported. 
1 Table 7. Performance (\%) on the BabyCare dataset for the MEABSA task, Accuracy, Marco-Precision, 2 Macro-Recall and Marco-F1 are reported.

\begin{tabular}{|l|l|l|l|l|}
\hline \multicolumn{1}{|c|}{ Models } & \multicolumn{1}{c|}{ Accuracy } & \multicolumn{1}{c|}{ Precision } & \multicolumn{1}{c|}{ Recall } & \multicolumn{1}{c|}{ F1 } \\
\hline MemNet & 62.74 & 59.81 & 48.84 & 46.13 \\
\hline ATAE-LSTM & 66.09 & 58.47 & 49.68 & 47.75 \\
\hline IAN & 61.93 & 41.71 & 47.04 & 43.73 \\
\hline MemNet+ & 65.32 & 59.93 & 50.55 & 47.93 \\
\hline ATAE-LSTM+ & 66.25 & 56.01 & 51.93 & 51.87 \\
\hline IAN+ & 65.81 & 44.42 & 50.06 & 46.50 \\
\hline CEA & 80.20 & 77.68 & 75.23 & 76.29 \\
\hline DT-CEA & 81.74 & - & - & 78.23 \\
\hline CADMN & 81.45 & - & - & 78.37 \\
\hline PEA(Our) & $\mathbf{8 5 . 7 2}$ & $\mathbf{8 3 . 9 7}$ & $\mathbf{8 2 . 6 0}$ & $\mathbf{8 3 . 2 5}$ \\
\hline
\end{tabular}




\section{Table 8 (on next page)}

Table 8. $p$-value between PEA and other baselines on ABSA, TABSA and MEABSA tasks. 
Table 8. $p$-value between PEA and other baselines on ABSA, TABSA and MEABSA tasks.

\begin{tabular}{|c|c|c|}
\hline \multicolumn{3}{|c|}{ ABSA Task } \\
\hline Dataset & Restaurant & Laptop \\
\hline TD-LSTM & $1.4379 \mathrm{e}-14$ & $1.4331 \mathrm{e}-14$ \\
\hline MemNet & $1.6116 \mathrm{e}-11$ & $1.7323 \mathrm{e}-09$ \\
\hline ATAE-LSTM & $2.0494 \mathrm{e}-16$ & $1.1331 \mathrm{e}-12$ \\
\hline IAN & $5.8819 \mathrm{e}-13$ & $1.2102 \mathrm{e}-12$ \\
\hline RAM & $9.4895 \mathrm{e}-13$ & $2.1595 \mathrm{e}-09$ \\
\hline TransCap & $1.1872 \mathrm{e}-06$ & 0.0138 \\
\hline ASGCN & $7.3462 \mathrm{e}-16$ & $3.7338 \mathrm{e}-07$ \\
\hline \multicolumn{3}{|c|}{ TABSA Task } \\
\hline Dataset & \multicolumn{2}{|c|}{ SentiHood } \\
\hline Dmu-Entnet & \multicolumn{2}{|l|}{$6.7790 \mathrm{e}-41$} \\
\hline BERT-pair-NLI-M & \multicolumn{2}{|l|}{0.0174} \\
\hline \multicolumn{3}{|c|}{ MEABSA Task } \\
\hline Dataset & \multicolumn{2}{|c|}{ BabyCare } \\
\hline MemNet & \multicolumn{2}{|l|}{$6.6475 \mathrm{e}-140$} \\
\hline ATAE-LSTM & \multicolumn{2}{|l|}{$8.3216 \mathrm{e}-113$} \\
\hline IAN & \multicolumn{2}{|l|}{$7.1802 \mathrm{e}-148$} \\
\hline MemNet + & \multicolumn{2}{|l|}{$3.4485 \mathrm{e}-120$} \\
\hline ATAE-LSTM+ & \multicolumn{2}{|l|}{$1.1143 \mathrm{e}-114$} \\
\hline IAN+ & \multicolumn{2}{|l|}{$1.6552 \mathrm{e}-114$} \\
\hline CEA & \multicolumn{2}{|l|}{$2.2666 \mathrm{e}-20$} \\
\hline
\end{tabular}




\section{Table 9 (on next page)}

Table 9. Performance (\%) of ablation study on 4 datasets. 
1

Table 9. Performance (\%) of ablation study on 4 datasets.

\begin{tabular}{|l|l|c|l|l|l|l|l|c|}
\hline \multirow{2}{*}{ Ablation } & \multicolumn{2}{|c|}{ BabyCare } & \multicolumn{2}{c|}{ SentiHood } & \multicolumn{2}{c|}{ Restaurant } & \multicolumn{2}{c|}{ Laptop } \\
\cline { 2 - 10 } & Accuracy & F1 & Accuracy & AUC & Accuracy & F1 & Accuracy & F1 \\
\hline CEA & 80.20 & 76.29 & 90.3 & 93.2 & 78.13 & 67.96 & 71.41 & 67.05 \\
\hline CEA+ER & 80.78 & 77.33 & 91.1 & 93.3 & - & - & - & - \\
\hline CEA+ER+NI & 81.06 & 77.55 & 91.3 & 94.0 & 79.45 & 70.31 & 71.83 & 67.22 \\
\hline BERT-based model & 84.12 & 81.62 & 93.8 & 97.1 & 83.52 & 76.11 & 76.99 & 72.40 \\
\hline PEA (our) & $\mathbf{8 5 . 7 2}$ & $\mathbf{8 3 . 9 7}$ & $\mathbf{9 4 . 3}$ & $\mathbf{9 7 . 4}$ & $\mathbf{8 4 . 8 2}$ & $\mathbf{7 8 . 1 4}$ & $\mathbf{7 8 . 6 8}$ & $\mathbf{7 5 . 0 7}$ \\
\hline
\end{tabular}

2 


\section{Table $\mathbf{1 0}$ (on next page)}

Table 10. Case study on misclassifications of BERT-based and data augmented CEA model. The straight underlined words are entity terms and the wavy underlined words are aspect terms. 
1 Table 10. Case study on misclassifications of BERT-based and data augmented CEA model. The straight underlined words are entity terms and the wavy underlined words are aspect terms.

\begin{tabular}{|c|c|c|c|}
\hline & Example 1 & Example 2 & Example 3 \\
\hline Input & $\begin{array}{l}\text { I tried } \underline{\text { Pampers }} \text { and } \\
\text { Inherent. I dislike the } \\
\text { smell of the former, and } \\
\text { the latter leaks. }\end{array}$ & $\begin{array}{l}\text { QiAn feels thin. I have } \\
\text { brought BobDog, it will } \\
\text { be delivered home. }\end{array}$ & $\begin{array}{l}\text { I am too poor to afford } \\
\underline{\text { Kao. My son and }} \\
\text { daughter are using } \\
\text { XWW, it is cheap. }\end{array}$ \\
\hline Entity & Pampers & BobDog & Kao \\
\hline Aspect & Anti-leakage & Thickness & Cost \\
\hline $\begin{array}{l}\text { BERT-based } \\
\text { model output }\end{array}$ & Negative & Neutral & Neutral \\
\hline $\begin{array}{l}\text { Data augmented } \\
\text { CEA output }\end{array}$ & Neutral & Positive & Neutral \\
\hline PEA output & Neutral & Neutral & Neutral \\
\hline Gold output & Neutral & Neutral & Negative \\
\hline
\end{tabular}




\section{Table $\mathbf{1 1}$ (on next page)}

Table 11. Macro-F1 and standard deviation of Macro-F1 (in the brackets) on evident polarity biased (EPB) test set and original test set. "DA" is short for Data Augmentation. 
1 Table 11. Macro-F1 and standard deviation of Macro-F1 (in the brackets) on evident polarity biased (EPB)

2 test set and original test set. "DA" is short for Data Augmentation.

\begin{tabular}{|l|l|l|l|}
\hline \multicolumn{1}{|c|}{ Models } & \multicolumn{1}{|c|}{ EPB test set } & \multicolumn{1}{c|}{ Original test set } & \multicolumn{1}{c|}{ Decline on EPB } \\
\hline CEA & $0.7542(0.0123)$ & $0.7714(0.0040)$ & $1.72 \%$ \\
\hline CEA+DA & $0.7753(0.0069)$ & $0.7768(0.0036)$ & $0.15 \%$ \\
\hline $\begin{array}{l}\text { BERT-based } \\
\text { model }\end{array}$ & $0.8068(0.0070)$ & $0.8162(0.0040)$ & $0.94 \%$ \\
\hline PEA & $0.8153(0.0069)$ & $0.8234(0.0061)$ & $0.81 \%$ \\
\hline
\end{tabular}

3 
Figure 1

Figure 1. The graphical abstract of the PEA model.

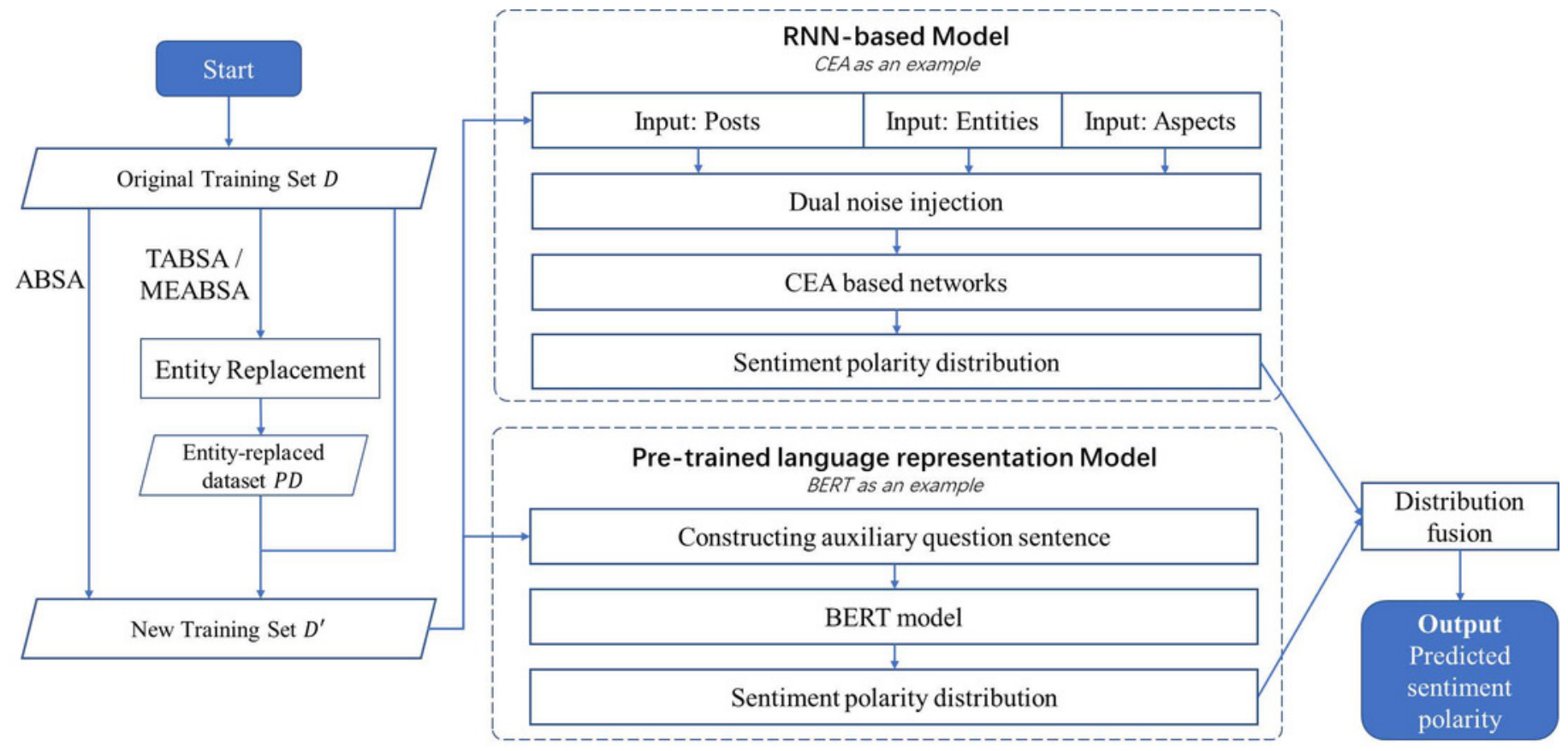


Figure 2

Figure 2. An example of dual noise injection.

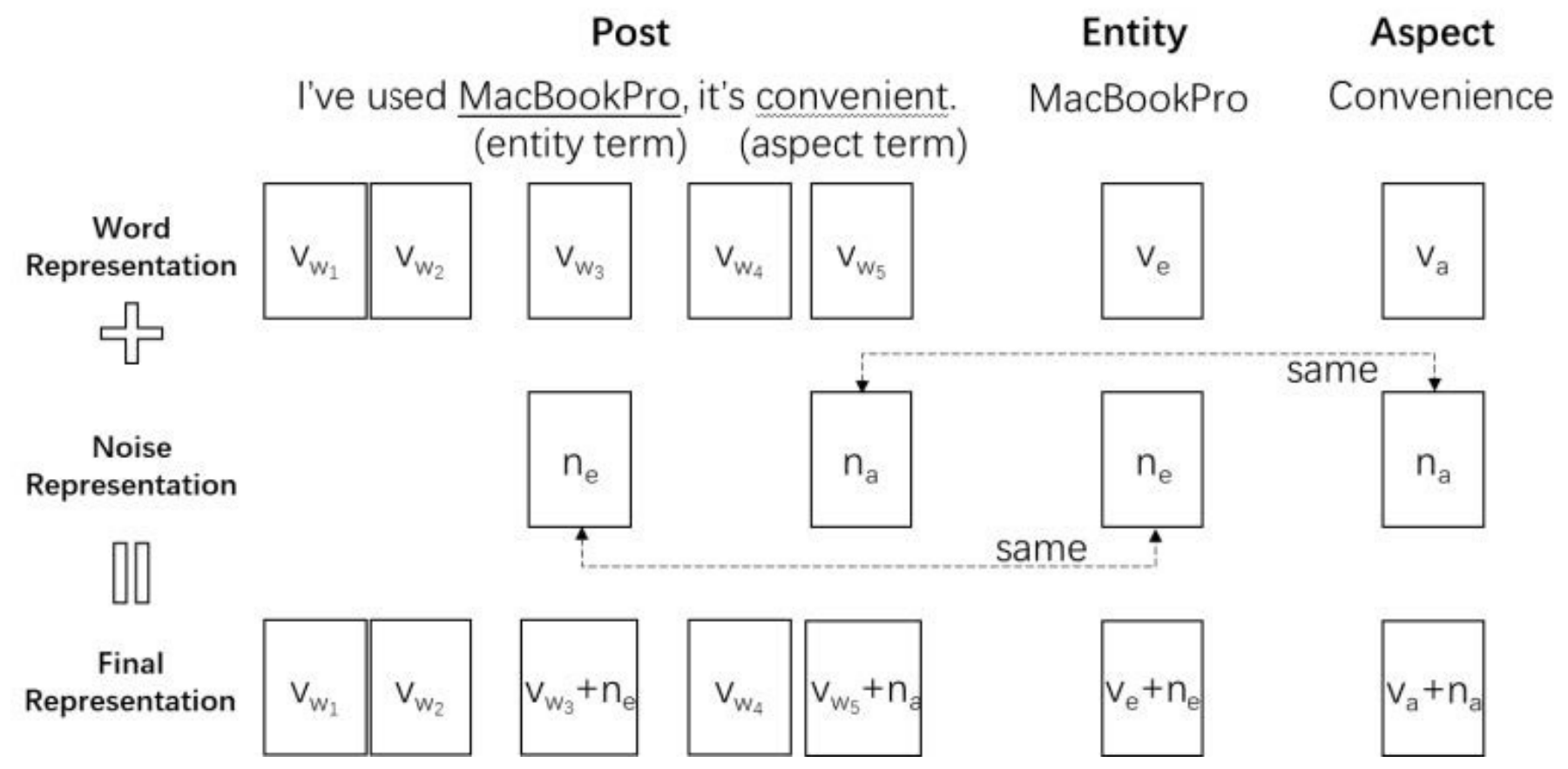


Figure 3

Figure 3. General structure of CEA with noise-injected vectors

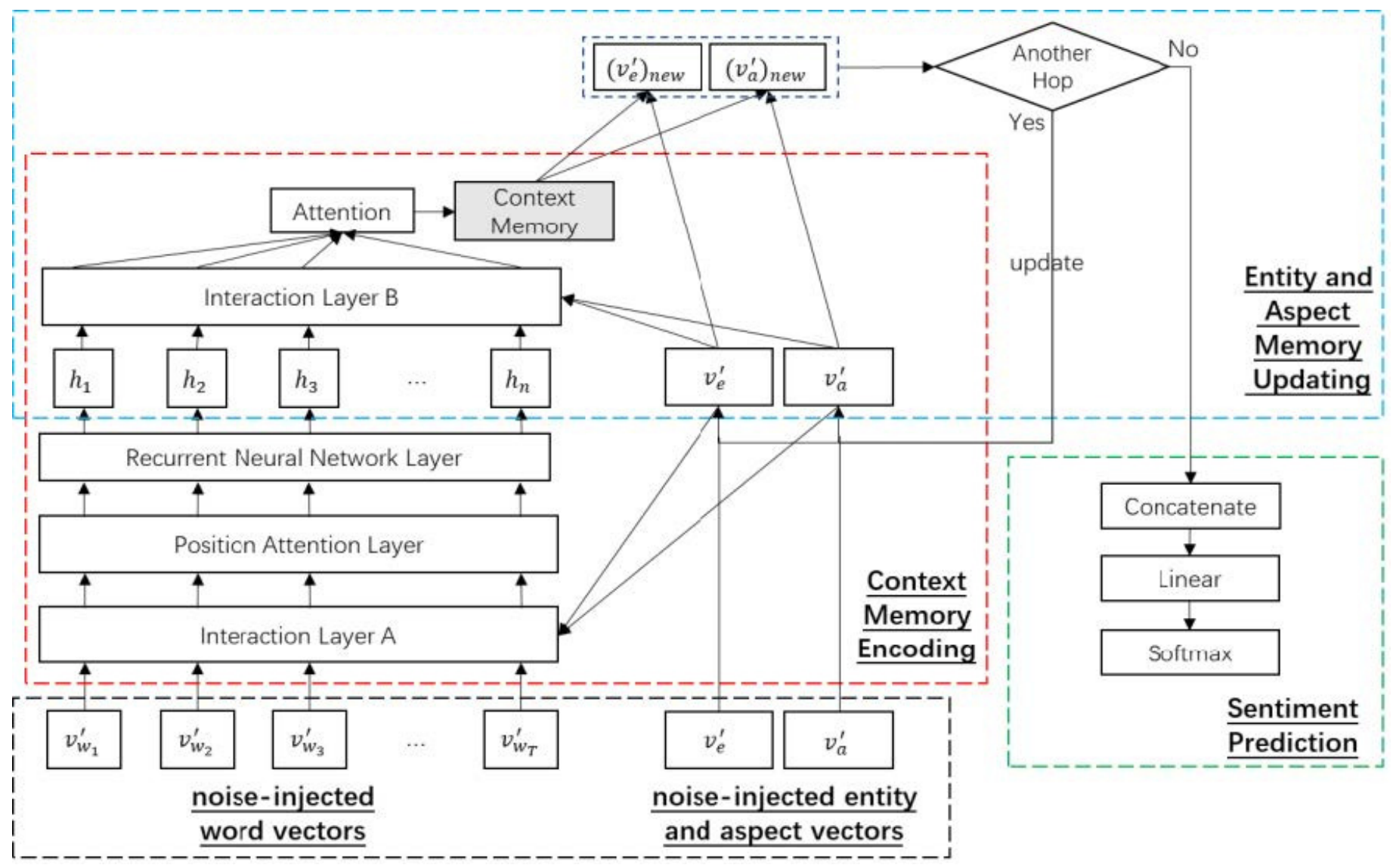


Figure 4

Figure 4. Macro-F1 performance on four datasets with different values of $\sigma$ in noise infection

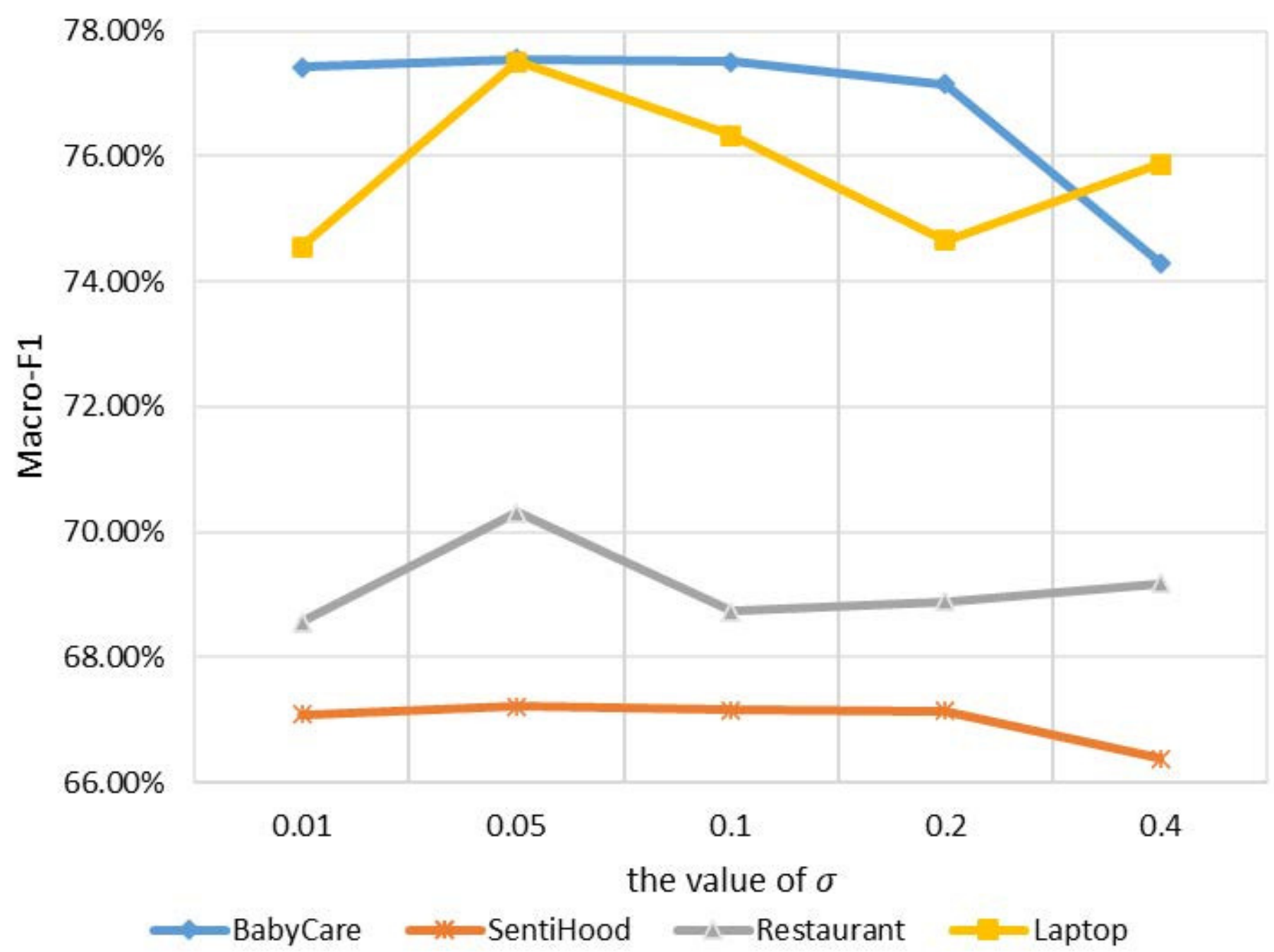


Figure 5

Figure 5. Box plot of the number of instances for every entity based on the original training and the entity-replaced dataset respectively.

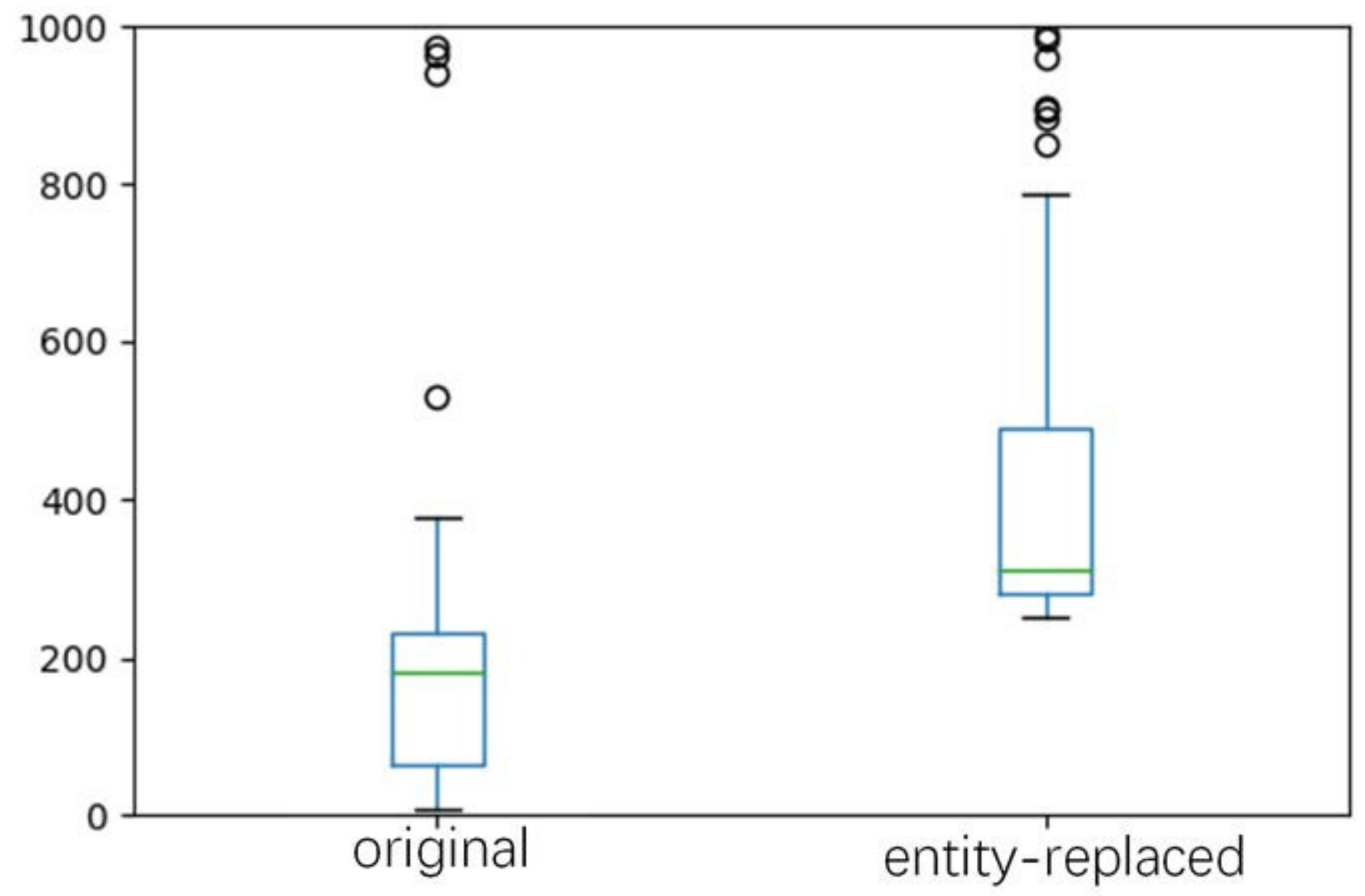


Figure 6

Figure 6. Performance on extreme low-resource conditions.

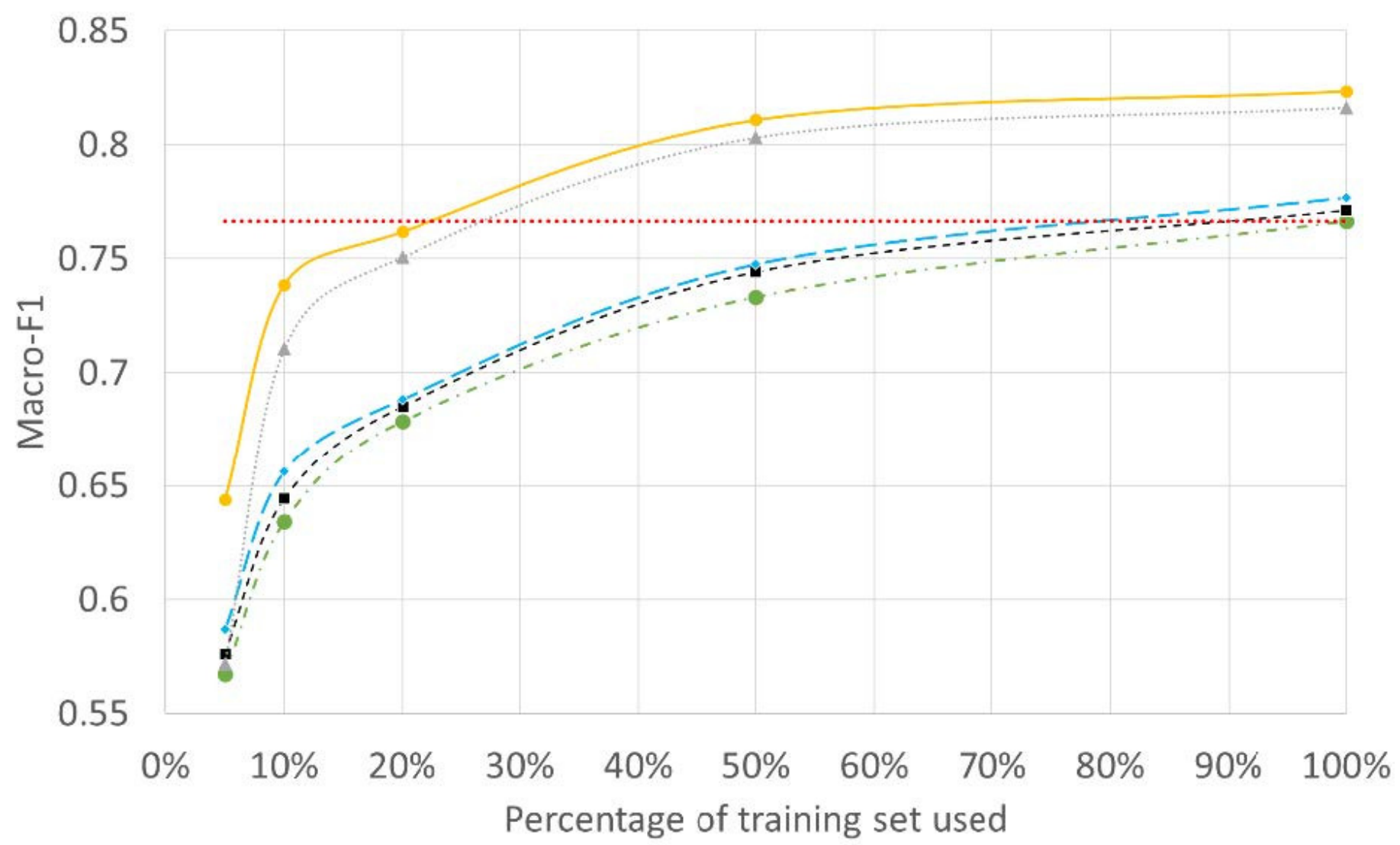

$\rightarrow-C E A \quad-\mathbf{m}$-CEA+ER $\rightarrow-$ CEA+ER+NI $\cdots$ - BERT-based model $\rightarrow$ PEA $\cdots \cdots$ Baseline: CEA with $100 \%$ data 